University of New Hampshire

University of New Hampshire Scholars' Repository

2-1986

\title{
Beyond bathymetry: Mapping acoustic backscattering from the deep seafloor with Sea Beam
}

Christian de Moustier

University of California - San Diego

Follow this and additional works at: https://scholars.unh.edu/ccom

Part of the Oceanography and Atmospheric Sciences and Meteorology Commons

\section{Recommended Citation}

de Moustier, Christian, "Beyond bathymetry: Mapping acoustic backscattering from the deep seafloor with Sea Beam" (1986). Journal of the Acoustical Society of America. 894.

https://scholars.unh.edu/ccom/894

This Journal Article is brought to you for free and open access by the Center for Coastal and Ocean Mapping at University of New Hampshire Scholars' Repository. It has been accepted for inclusion in Center for Coastal and Ocean Mapping by an authorized administrator of University of New Hampshire Scholars' Repository. For more information, please contact Scholarly.Communication@unh.edu. 


\title{
Beyond bathymetry: Mapping acoustic backscattering from the deep seafloor with Sea Beam
}

\author{
Christian de Moustier \\ Marine Physical Laboratory, Scripps Institution of Oceanography, University of California, San Diego, \\ California 92093
}

(Received 10 June 1985; accepted for publication 15 October 1985)

\begin{abstract}
In its standard mode of operation, the multibeam echo sounder Sea Beam produces high resolution bathymetric contour charts of the seafloor surveyed. However, additional information about the nature of the seafloor can be extracted from the structure of the echo signals received by the system. Such signals have been recorded digitally over a variety of seafloor environments for which independent observations from bottom photographs or sidescan sonars were available. An attempt is made to relate the statistical properties of the bottom-backscattered sound field to the independently observed geological characteristics of the seafloor surveyed. Acoustic boundary mapping over flat areas is achieved by following trend changes in the acoustic data both along and across track. Such changes in the acoustics are found to correlate with changes in bottom type or roughness structure. The overall energy level of a partial angular-dependence function of backscattering appears to depend strongly on bottom type, whereas the shape of the function does not. Clues to the roughness structure of the bottom are obtained by relating the shape of the probability density function of normal-incidence echo envelopes to the degree of coherence in the backscattered acoustic field.
\end{abstract}

PACS numbers: 43.30.Bp, 43.30.Gv, 92.10.Vz, 91.50.Ey

\section{INTRODUCTION}

In the past few years, multibeam echo sounders have become available to the scientific community, allowing investigators to map, with high resolution and in near-real time, a large swath of seafloor on each traverse of the ship. The bathymetric charts thus obtained represent a great improvement over those drawn from conventional single-point depth recording systems. However, bathymetry reveals the shape of seafloor features only to the resolution of the sounding system; it does not yield other seafloor characteristics, such as bottom type or bottom microroughness and their respective lateral homogeneity.

Because bottom roughness and variations in bottom substrate cause fluctuations in the backscattered acoustic signal, such seafloor characteristics can be inferred, in part, by analyzing the structure and the variations of this signal over several transmission cycles (pings). This analysis is necessarily statistical, and deals with an ensemble of independent samples since, as the ship moves, each ping ensonifies a slightly different portion of seafloor. In order to relate the statistics of the backscattered sound field to the characteristics of the seafloor from which it emanates, two main theoretical approaches have been taken. By analogy with the Huygens-Fresnel principle of physical optics, ${ }^{1}$ both approaches consider that the sound field scattered by the seafloor consists of elementary waves in mutual phase interference. Their difference lies in the representation of the irregularities of the seafloor. In the first approach, the seafloor is a surface whose irregularities are described by a rootmean-square (rms) roughness in the vertical dimension, and a correlation distance or area in the lateral dimension. The statistics of the surface are then incorporated into the Helm-
holtz-Kirchhoff formulation of the scattering theory so that statistics of the sound field (usually a Gaussian process) can be used to estimate the characteristics of the surface..$^{2-8}$ The second approach describes the rough surface as a random distribution of point scatterers reradiating independently in time. It is the quasiphenomenological model of Faure, ${ }^{9}$ Ol'shevskii, ${ }^{10}$ and Middleton, ${ }^{11}$ which uses a Poisson process giving clues to the density of the scatterers. In this model, however, no relation exists between the statistics of the scatterers and those of the rough surface. For this reason, the first approach mentioned above is preferred in this paper.

In the Helmholtz-Kirchhoff formulation, it is possible to determine a measure of the degree of coherence in the backscattered sound field, and use it to relate the shape of the probability density function (pdf) of echo envelopes to the characteristics of the surface (rms roughness, correlation function).${ }^{12}$ Because of their inherent narrow-beam geometry, multibeam echo sounders are well suited for such analysis. In addition, backscatter measurements with well-defined spatial resolution are obtainable both along and across the ship's track. A description of seafloor characteristics is then possible in terms of acoustic boundaries.

This paper presents the results of an experiment to map seafloor acoustic backscattering boundaries with a Sea Beam multibeam echo sounder operating at a frequency of $12 \mathrm{kHz}$ with a 7-ms pulse length and a $22 \mathrm{deg}$ angular resolution. As this system does not retain the acoustic data it receives, a parallel data acquisition system, ${ }^{13}$ built by the Marine Physical Laboratory (MPL) of the Scripps Institution of Oceanography, was used to record digitally the envelope of the detected echoes over a variety of seafloor environments. These data are analyzed as follows: 
(1) First-order statistics (mean and variance) of peak amplitude in the near-specular direction, and of total energy for nonspecular beams serve to quantify spatial homogeneity of the backscattered sound field.

(2) Angular dependence of total energy is used to complement (1) in the estimation of acoustic boundaries over the swath of seafloor ensonified by the Sea Beam.

(3) Probability density functions of peak amplitude in the near-specular direction are compared to a Rician pdf to estimate the degree of coherency of the backscattered signals, yielding a qualitative estimate of the roughness structure of the seafloor.

However, the lack of phase information, occasional saturation in both the data acquisition and the Sea Beam systems, and sidelobe interference problems limit the scope of this analysis. Therefore, this paper only intends to show the potential for determining seafloor characteristics that exist in acoustic backscatter measurements derived from a multibeam echo sounder. Also, acoustic measurements alone are insufficient to determine the exact nature of the bottom. Consequently, ground truth must be obtained by independent remote sensing (e.g., deep-sea photography and/or television, core or grab samples, etc.). To this end, most of the data presented here are supported by at least one independent source of measurements.

\section{THEORY}

In this section, we first consider the applicability of the Helmholtz-Kirchhoff formulation for seafloor acoustic backscattering to backscatter measurements made with a Sea Beam system. Next, we give an expression which relates the degree of coherence of the backscattered sound field to the roughness and correlation structure of the scattering surface. As shown by Stanton, ${ }^{12}$ an estimate of these parameters is then obtainable from the shape of the pdf of normal-incidence echo envelopes.

\section{A. Seafloor acoustic backscattering}

When measuring acoustic backscatter from the deep seafloor, the first common observation is that individual echoes are not reproducible and that there can be several $\mathrm{dB}$ of variation in the amplitude of the returned signal from one ping to the next. The backscattering process is therefore considered stochastic, and its description needs to be statistical.

Expressions for the sound pressure of a bottom echo impinging upon a hydrophone array have been derived ${ }^{4-8}$ using the Helmholtz-Kirchhoff formulation in the bistatic scattering from a rough surface. In the backscattering case, omitting the time dependence and propagation losses, a receiver at $Q$ (Fig. 1) will sense the pressure:

$$
\begin{aligned}
p(Q)= & j B_{1} \exp \left(2 j \kappa R_{0}\right) \int_{-\infty}^{+\infty} \int_{0}^{\infty} \exp [2 j(a x+c \xi \\
& \left.\left.+\frac{\kappa}{2 R_{0}}\left(x^{2} \cos ^{2} \theta+y^{2}\right)\right)\right] d x d y
\end{aligned}
$$

with

$a=\kappa \sin \theta, \quad c=-\kappa \cos \theta, \quad B_{1}=\kappa B \mathbf{R} / 2 \pi \mathbf{R}_{0}^{2} \cos \theta$.

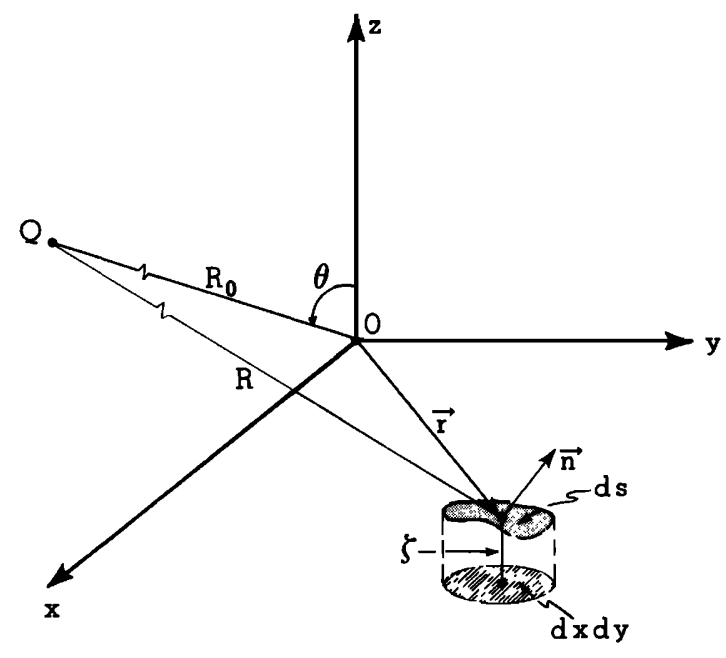

FIG. 1. Geometry of backscattering. Source and receiver are at $Q(x, z)$, a distance $R$ away from the element of surface $d s$. Here, $\zeta(x, y)$ is the elevation of $d s$ above the $x y$ plane.

The notation follows that of Clay and Medwin (Ref. 6, Appendix 10 ), where $B$ is proportional to the source power, $D_{0}$ is the source directivity, $\kappa$ is the acoustic wavenumber, and $\mathbf{R}$ is the reflection coefficient. As shown in Fig. $1, R_{0}$ is the range to the center of the scattering region and $\theta$ is the angle of incidence. In their notation, $\theta=\theta_{1}$ with $\theta_{2}=-\theta_{1}$ and $\theta_{3}=0$, while $a$ and $c$ are the horizontal and vertical components of wavenumber. ${ }^{14}$ Also, $\zeta$ is the normal deviation of the scattering surface from the $x y$ plane and depends on position in the plane: $\zeta=\zeta(x, y)$; it characterizes the roughness of the surface. In the limit $\zeta=0$, corresponding to a plane surface, the return is a specular reflection at normal incidence, and outside of normal incidence, Eq. (1) is a function of the beam pattern of the acoustic array. At the other extreme, when $\zeta$ is large, the return comes mostly from acoustic energy scattered by the rough surface back towards the receiver. So, in the general case, the return is a combination of scattered (incoherent) and reflected (coherent) energy which varies depending on the angle of incidence and the beam pattern of the array.

Equation (1) is derived in the farfield of the transmit/ receive system so that ranges $R$ can be approximated by $R_{0}$ (Fig. 1) in the expressions of the incident and backscattered pressure fields, except in the phase terms, where $R$ is expanded to second-order terms to account for Fresnel zone contributions. This approximation is valid for Sea Beam's 12-cm wavelength at ocean depths.

The Kirchhoff approximation is also used. It assumes that in the boundary conditions on the surface, the reflection coefficient $\mathbf{R}$ can be used at every point on the surface by approximating the field at any point on the surface by that which would be present on the tangent plane at that point (Ref. 3, Chap. 3). This requires that the radius of curvature of the irregularities on the seafloor be large compared to the acoustic wavelength or that no shadowing effects occur within the ensonified area. These conditions are met in most of the data presented here.

Equation (1) also assumes that the area ensonified is small compared to $R_{0}$, so that the dependence of $R_{0}$ on $x, y$, 
or $\zeta$ can be ignored. Likewise, $\mathbf{R}, a$, and $c$, which depend mostly on the angle of incidence $\theta$, are assumed to have only small variations within the scattering area. These approximations therefore require that the acoustic system have a small beam width and commensurate pulse length. Both assumptions are reasonable for the Sea Beam system.

It follows that, for a given angle of incidence $\theta$, the random character of $p(Q)$ in Eq. (1) is mostly due to the fluctuations of the phase term $e^{2 j c 5}$, which accounts for the irregularities of the bottom. As a consequence, the bottom roughness $\zeta$ is considered a random process, and a statistical description of the backscattered sound field can be achieved by ensemble averaging returns from successive pings. Such returns are independent from each other since the ship moves between pings and a slightly different portion of seafloor is sampled every time. For an ensemble of such surfaces over several pings, the stochastic function $\zeta(x, y)$ is characterized by an rms roughness amplitude (assuming $\zeta$ has a zero mean):

$$
\sigma=\left\langle\zeta(x, y)^{2}\right\rangle^{1 / 2},
$$

a "correlation function,"

$$
C\left(x^{\prime}, y^{\prime}\right)=\left(1 / \sigma^{2}\right)\left\langle\zeta(x, y) \zeta\left(x+x^{\prime}, y+y^{\prime}\right)\right\rangle,
$$

and a pdf $W(\xi)$. Equations (2) and (3) describe, respectively, the vertical and the lateral dimensions of the random rough surface.

Assuming $\zeta$ to be normally distributed with zero mean, the (ensemble) average pressure $\langle p\rangle$ is related to the degree of coherence in the backscattered sound, ${ }^{2,4}$ and an estimate of the rms roughness $\sigma$ is directly obtainable from measurements of the coherently backscattered signals $\langle p\rangle$. However, although this is a proven technique, ${ }^{4,5,15,16}$ it requires measurements of the phases of the echo signal, which are not available in our data. For this reason, we resort to the meansquare pressure:

$$
\left\langle p^{2}\right\rangle=\left\langle p p^{*}\right\rangle,
$$

where $p^{*}$ is the complex conjugate of $p$.

As summarized in Appendix A, the mean-square pressure $\left\langle p^{2}\right\rangle$, which includes the contribution of both the reflected (coherent) and scattered (incoherent) components of the backscatter, is related to the statistics of the rough surface through the rms roughness $\sigma$ and the spatial correlation function $C(\xi, \eta)$. To estimate these parameters requires the determination of the respective contributions from the coherent and incoherent components to the total backscattered field, which is equivalent to obtaining a measure of the degree of coherence in the field. To this end, the total mean-square pressure is expressed as the sum of the coherent $\langle p\rangle^{2}$ and incoherent $\left\langle S^{2}\right\rangle$ components (Ref. 4, p. 204),

$$
\left\langle p^{2}\right\rangle=\langle p\rangle^{2}+\left\langle S^{2}\right\rangle,
$$

where $\langle p\rangle^{2}=\langle p\rangle\left\langle p^{*}\right\rangle$, so that $\left\langle S^{2}\right\rangle$ is simply the meansquare second moment of $p$. It follows that the ratio of the coherent to the incoherent components,

$$
\gamma=\langle p\rangle^{2} /\left\langle S^{2}\right\rangle,
$$

is a measure of the degree of coherence of the backscattered field. Other authors ${ }^{17}$ have used the ratio of the coherent part $\langle p\rangle^{2}$ to the total mean-square pressure $\left\langle p^{2}\right\rangle$ as a mea- sure of the degree of coherence. Both expressions are valid, but Eq. (6) is the more useful in this context since it gives a link between the statistics of the backscattered field and a Rician pdf. ${ }^{12}$

At normal incidence $(\theta=0), \gamma$ is related to the parameters of the rough surface $\sigma$ and $C$ by

$$
\begin{aligned}
\gamma^{-1}= & \frac{\sqrt{\alpha \beta}}{\pi} \int_{-\infty}^{+\infty} \exp \left[-\left(\alpha \xi^{2}+\beta \eta^{2}\right)\right] \\
& \times\left\{\exp \left[4 \kappa^{2} \sigma^{2} C(\xi, \eta)\right]-1\right\} d \xi d \eta,
\end{aligned}
$$

where $\alpha$ and $\beta$ account for the acoustic geometry of the measuring system [Appendix A, Eq. (A7)]. To integrate Eq. (7) requires the determination of an analytical form of the correlation function. Although several forms of this function have been used in rough-surface scattering theory, ${ }^{18}$ most of them are one dimensional and are restricted to ripplelike features. Stanton ${ }^{12}$ introduced a correlation area by considering a two-dimensional correlation function which is zero on the average except for a small "elliptic cone" around $\xi=\eta=0$, where it decreases linearly from 1 at $\xi=\eta=0$. His application was a surface consisting of ripples, but the approach is also valid for other types of roughness (nodule fields, bed rocks, etc. ), more representative of our data. For ease of integration, we prefer to use, as a two-dimensional correlation function, an elliptic paraboloid such that

$$
C(\xi, \eta)= \begin{cases}1-\left(\xi^{2} / l_{1}^{2}\right)-\left(\eta^{2} / l_{2}^{2}\right), & \geqslant 0, \\ 0, & \text { otherwise. }\end{cases}
$$

In the limit $\left(4 \kappa^{2} \sigma^{2}<1\right)$ of small to moderate roughness, the second exponential in Eq. (7) is expandable in a Taylor series. Keeping the first two terms of the expansion and using Eq. (8), Eq. (7) integrates into

$$
\gamma^{-1} \simeq(16 / 3)(\sqrt{\alpha \beta} / \pi) \kappa^{2} \sigma^{2} l_{1} l_{2},
$$

where $l_{1}$ and $l_{2}$ are the correlation distances beyond which $C(\xi, \eta)=0$.

It has been assumed that for $|\xi| \leqslant l_{1}$ and $|\eta| \leqslant l_{2}$ the contribution of the first exponential term in Eq. (7) can be neglected. This is equivalent to requiring the exponential term to reach $e^{-1}$ outside of the range $|\xi| \leqslant l_{1}|\eta| \leqslant l_{2}$, so that $\alpha \xi^{2}+\beta \eta^{2}=1$ for $|\xi|>l_{1}$ and $|\xi|>l_{2}$. Except for a factor of 2, Eq. (9) is equivalent to Stanton's ${ }^{12} \mathrm{Eq}$. (16). It shows that for small to moderate roughness, the degree of coherence in the specular backscattered sound field depends on the relative roughness $(\kappa \sigma)$ and the correlation area $\left(l_{1} l_{2}\right)$ of the surface, as well as on the beam widths of the measuring system $(\alpha, \beta)$. Because the same degree of coherence is a parameter which controls the shape of the pdf of normal incidence echo envelopes, Eq. (9) makes it possible to evaluate the parameters of the rough surface directly from the shape of the pdf. Also, pending determination of the rms roughness from coherent measurements, the correlation area is readily obtained from Eq. (9).

\section{B. Envelope distributions}

As summarized in Appendix B, the Rician distribution ${ }^{19}$ is useful in describing the effects of coherent scattering on the shape of the pdf of echo envelopes. By considering the 
backscattered pressure wave as a sum of a coherent component of rms value $A$ and an incoherent component normally distributed with variance $\sigma_{p}^{2}$, the parameter $\gamma$ can be written as

$$
\gamma=A^{2} / \sigma_{p}^{2}
$$

It follows that the Rician distribution for the corresponding echo envelopes $E$ can be expressed as a function of $E$, its second moment $\left\langle E^{2}\right\rangle$, and $\gamma$ :

$$
\begin{aligned}
W(E)= & (2+\gamma) \frac{E}{\left\langle E^{2}\right\rangle} \exp \left(-\frac{(2+\gamma) E^{2}+\gamma\left\langle E^{2}\right\rangle}{2\left\langle E^{2}\right\rangle}\right) \\
& \times I_{0}\left\{\left(E /\left\langle E^{2}\right\rangle^{1 / 2}\right)[\gamma(2+\gamma)]^{1 / 2}\right\} .
\end{aligned}
$$

In the limit where $\gamma$ tends to zero (dominance of the incoherent component), Eq. (11) tends to the Rayleigh distribution [Eq. (B4) ]. Conversely, when the coherent component dominates $(\gamma>1)$, Eq. (11) tends to Gaussian-like distribution. A qualitative estimate of the degree of coherence in the backscattered sound field can then be obtained directly from the value of $\gamma$ that gives the best fit of the Rician distribution to a histogram of the normal-incidence echo-envelope peak distribution.

This is analogous to the determination of the contrast of a speckle pattern ${ }^{20}$ in laser optics. Speckle patterns are the result of constructive and destructive interference between light waves scattered from elementary areas of a rough surface. Their contrast is analogous to $\gamma^{-1}$ [Eq. (7)] since an ideal mirror surface does not produce a speckle pattern (contrast $=0$ ).

As in optics, useful quantitative results concerning the roughness structure of the surface are obtained for acoustic backscatter only when the roughness of the surface is a fraction of the acoustic wavelength. In such cases, the value of $\gamma$ derived from the shape of the pdf can be directly related to the product of the rms roughness and correlation area of the surface [Eq. (9) ]. For practical purposes, this technique is applicable when $\gamma>1$ because there is little difference in the shape of the pdf's for $0<\gamma<1$ (e.g., Ref. 12, Fig. 2).

\section{SEA BEAM ACOUSTIC DATA}

To explain some of the peculiarities of the Sea Beam data presented here, a brief description of the acoustic geometry is necessary.

\section{A. Acoustlc geometry}

Sea Beam uses two arrays perpendicular to each other. The transmit array consists of 20 projectors aligned along the ship's keel. The outputs of the projectors are amplitude shaded for sidelobe control according to the Dolph-Chebyschev method, ${ }^{21}$ and phase corrected to ensure vertical projection at all times. The resulting beam pattern is $22 \mathrm{deg}$ wide in the fore-aft direction and $54 \mathrm{deg}$ athwartships [Fig. $2(a)]$. These angles correspond to the half-power point of

(a) TRANSMIT

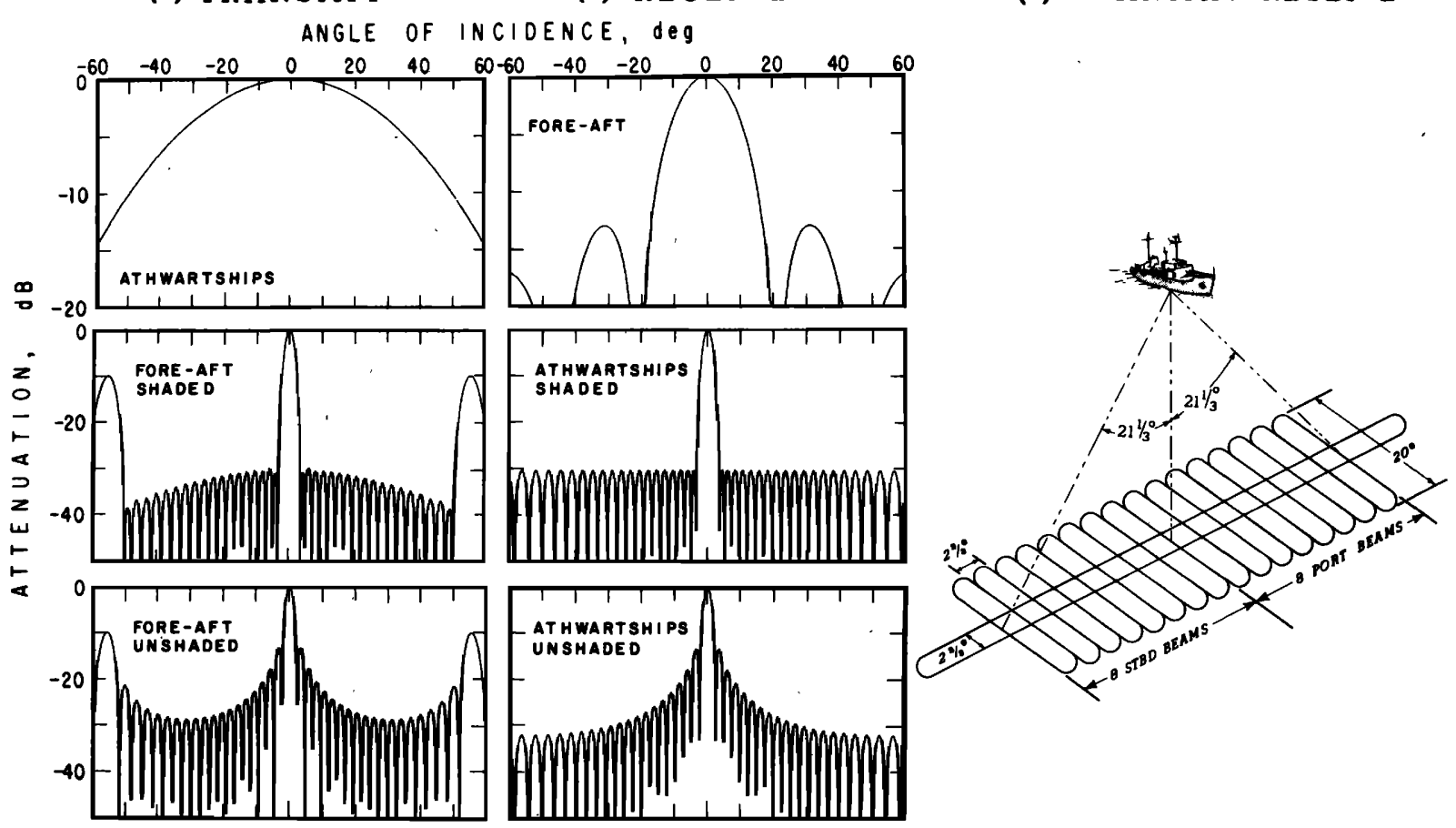

FIG. 2. Sea Beam transmit/receive geometry. Theoretical beam pattern cross sections are computed in the athwartships vertical plane centered on the array and in the vertical plane through the ship's fore-aft axis. The transmit beam pattern (a) spans 54 deg athwartships by 22 deg in the fore-aft direction. The receive beam pattern (b) is $20 \mathrm{deg}$ wide in the fore-aft direction by $22 \mathrm{deg}$ athwartships. Sixteen such beams are formed by steering the receiving hydrophone array athwartships between $\pm 20 \mathrm{deg}$ of incidence at $22 \mathrm{deg}$ intervals. The effect of Dolph-Chebyschev amplitude shading is shown for both arrays with Sea Beam's design criterion of 30-dB sidelobe attenuation. A cartoon (c) shows the angular relationship between the mainlobes of the transmit and receive beam patterns. Their intersection is represented by 16 "squares" 223 deg on a side. 


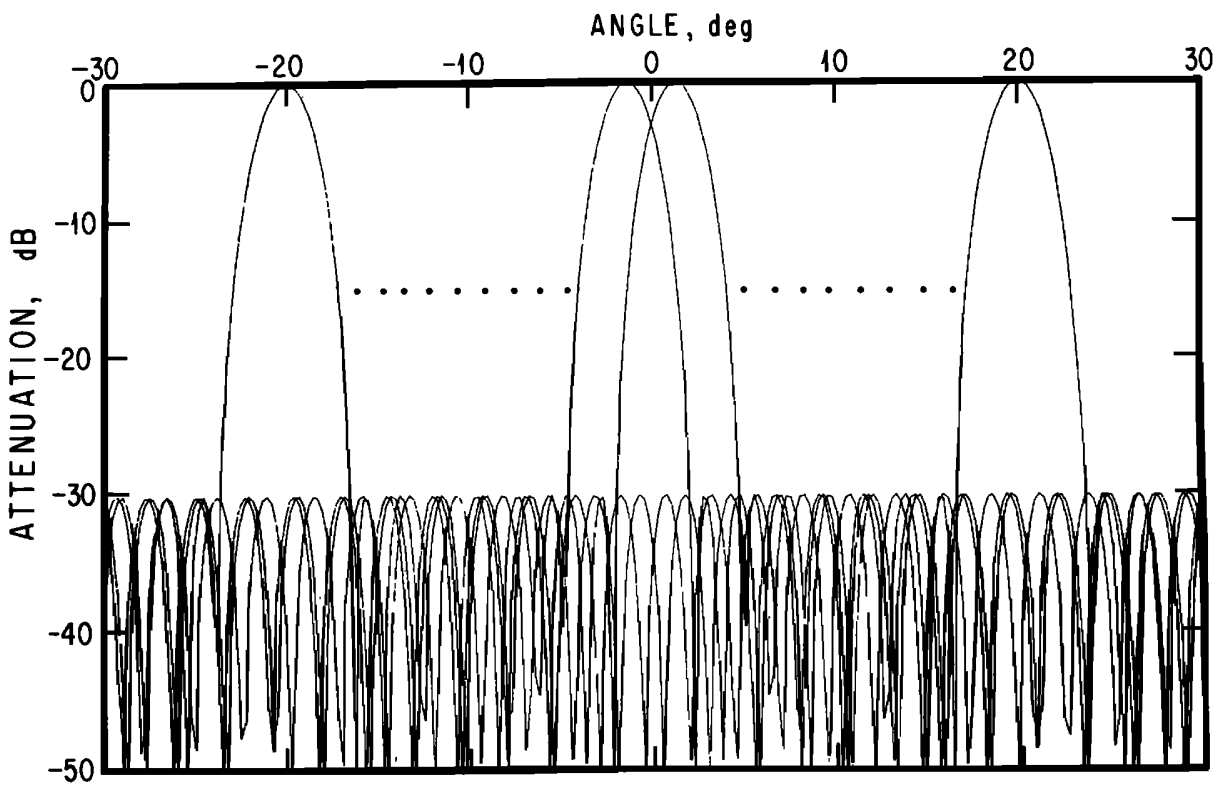

FIG. 3. Preformed beams. Four out of the sixteen preformed beams are displayed to show how sidelobes from each beam "look" into the direction of the mainlobe of all the others. The sidelobe contributions to the 16 bottom echoes received each ping can be seen in Fig. 4.

the beam patterns. This convention will be kept in the following.

The receive array consists of 40 elements arranged athwartships in a V-shaped array symmetrical about the ship's keel. The outputs of these elements are amplitude shaded (Dolph-Chebyschev shading) for sidelobe control, and the resulting beam is steered phasewise to form 16 preformed beams spaced $2 \frac{2}{3}$ deg apart between +20 and $-20 \mathrm{deg}$ of incidence. Each beam is $2 \frac{2}{3} \mathrm{deg}$ wide in the athwartships direction and 20 deg fore-aft [Fig. 2(b) ]. The comparatively wide beam width in the fore-aft direction is meant to accommodate pitch angles of $\pm 10 \mathrm{deg}$ for no pitch correction is performed on the receive array. Note also that each of the preformed beams has sidelobes pointing in the direction of (but not necessarily aligned with) the mainlobe of all the other beams (Fig. 3). As will be shown in the following, this fact is significant when dealing with echo envelopes of the 16 preformed beams. For a broader description of the Sea Beam system, the reader is referred to a comprehensive review by Renard and Allenou. ${ }^{22}$

\section{B. Sea Beam digltized echo envelopes}

Figure 4 illustrates a typical set of echo envelopes as they appear at the output of Sea Beam's echo-processor receivers. Each envelope corresponds to the return on one of the 16 preformed beams numbered 1-8 from the center of the ship out, on both port and starboard. These data have not been roll compensated; therefore, the ship's center line does not necessarily lie on the true vertical. The ridge of synchronous returns corresponds to energy from a strong return in

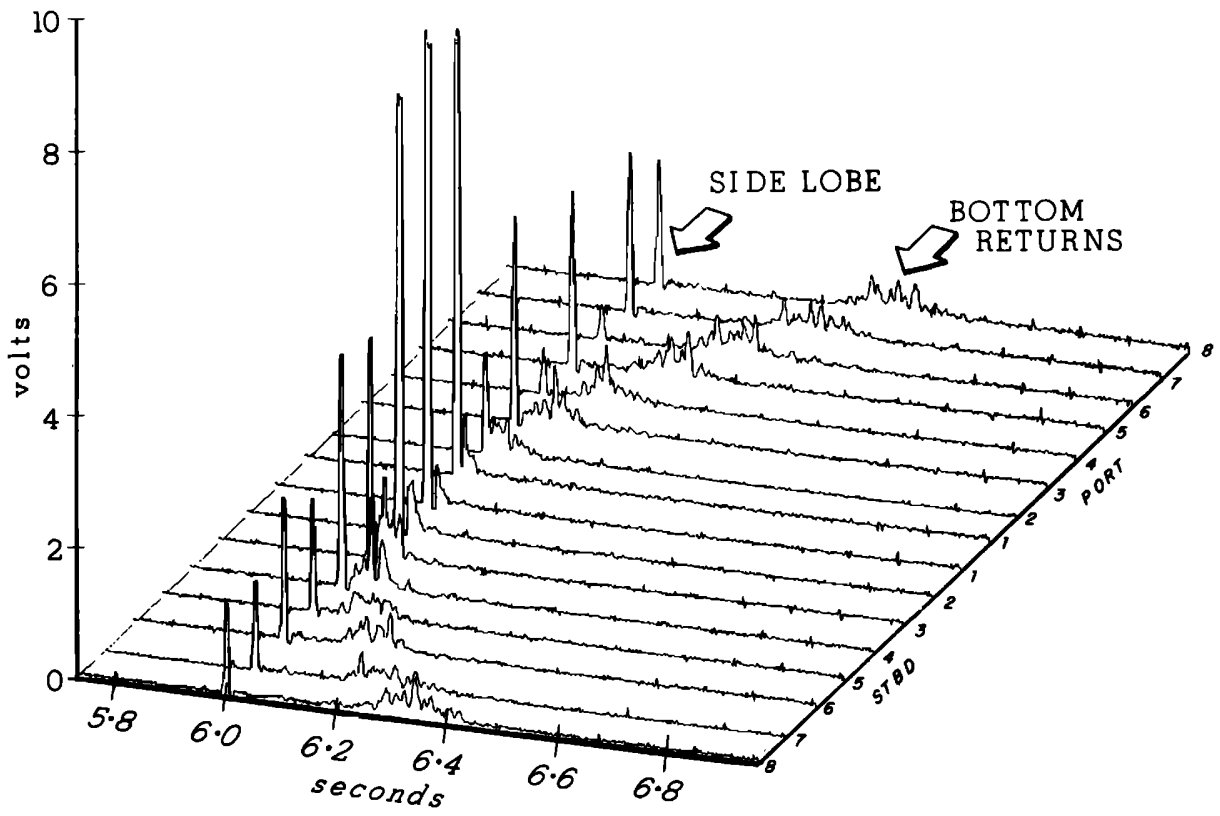

FIG. 4. Acoustic signal envelopes of the 16 preformed beams at the output of Sea Beam's echo processor receivers. Time is in seconds after transmission. Signal amplitude is in volts corrected for acoustic transmission loss by a time-varied gain. In this display, the data have not been corrected for ship roll or receiver gain. The ridge of synchronous returns is the sidelobe response to a strong return in the near-specular direction (STBD beam \#1 in this figure). 
the near-specular direction leaking into the sidelobes of all the other beams (e.g., starboard beam $\# 1$ in this figure). The term near-specular is used here to indicate that because Sea Beam receives with discrete beams, the return may be within $22 \mathrm{deg}$ of the specular direction. Likewise, in the following, nonspecular beams refer to those beams which are not within $22 \mathrm{deg}$ of the specular direction. In its current mode of operation, the Sea Beam echo processor digitizes these echo envelopes and applies ray bending, roll, gain, and sidelobe corrections. A time of arrival is then determined at the center of mass of each of the corrected echoes and is converted into a depth and a cross-track distance. These are, in turn, logged on magnetic tape, as well as recorded on paper as a bathymetric contour chart. No further use is made of the echo signals, which are then discarded. In an effort to preserve these echo signals for analysis, MPL built a parallel acoustic data acquisition system to record digitally data such as those of Fig. 4, along with a time-varied gain and the ship's roll angle. In this paper, some of the envelope data collected with the MPL system suffer amplitude clipping due to saturation in both the data acquisition system and the Sea Beam echoprocessor receivers. The saturation in the receivers occasionally disrupts Sea Beam's echo processing, resulting in spurious depth measurements. Likewise, because the echo processing is done on the envelope of the signals, the system cannot differentiate between sidelobe response and bottom return when the two overlap (e.g., beam \#2 in Fig. 4). Improper depth determination and bathymetric artifacts result. Such bathymetric artifacts have been analyzed in detail elsewhere, ${ }^{23}$ and we shall not repeat the discussion here. Nevertheless, this sidelobe interference constitutes a serious hindrance when analyzing signal envelopes where sidelobe and bottom return overlap. To tell them apart requires phase information which is not available in our present data set.

\section{Data reduction}

Owing to the limitations outlined above, the acoustic data recorded from Sea Beam were reduced to be analyzed in two ways. The first approach concentrates on the statistics of the peak amplitude in the near-specular direction; the sec- ond deals with both angular dependence and statistics of total energy in nonspecular beams. To overcome the saturation problem mentioned above, the mean sidelobe response was used to retrieve the peak amplitude of the near-specular returns that appeared clipped. The method assumes that the ratio of the near-specular peak amplitude to the corresponding mean sidelobe response is approximately constant from ping to ping, and that most of the variability in this ratio is due to (1) the slight misalignment of sidelobes with the mainlobe of the near-specular beam (Fig. 3), and (2) the roll of the ship. For simplicity, only data collected over nearly flat seafloor (slope angles less than $22 \mathrm{deg}$ ) were used in this exercise, since, owing to the acoustic geometry, no specular backscatter is to be expected from bottoms sloping up or down. To avoid bias from bottom returns, the mean sidelobe response was computed as the arithmetic mean of the peak values of sidelobe contributions well separated from the bottom returns. As an example, beams number port 8-3 and starboard 4-8 would qualify in Fig. 4. By working on nonclipped data, this mean was then compared with the amplitude of the corresponding near-specular peak by computing their ratio.

From an ensemble of over 400 such ratios the mainlobe to sidelobe ratio is $17.80(25 \mathrm{~dB})$ with a standard deviation of 2.14. This value is in agreement with what Dolph ${ }^{21}$ predicted for his amplitude shading method. It is, however, 2 to $3 \mathrm{~dB}$ lower than the level measured by Renard and Allenou ${ }^{22}$ (27-28 dB) on a different Sea Beam system. But, because their measurements were done on only two preformed beams, it is reasonable to expect the level obtained by averaging over ten beams to be lower.

The ping-to-ping variations of the near-specular peak were then inferred from the variations of the corresponding mean sidelobe response using the same method. When the sidelobe response had insufficient signal-to-noise ratio, the near-specular return was not clipped so its peak amplitude was used to compute the mean sidelobe level by subtracting $25 \mathrm{~dB}$. Results of this method are illustrated in Fig. 5.

Because of the time stretching evidenced on the nonspecular returns (Fig. 4), their description is more appropriately based on total energy than on peak amplitude. To es-
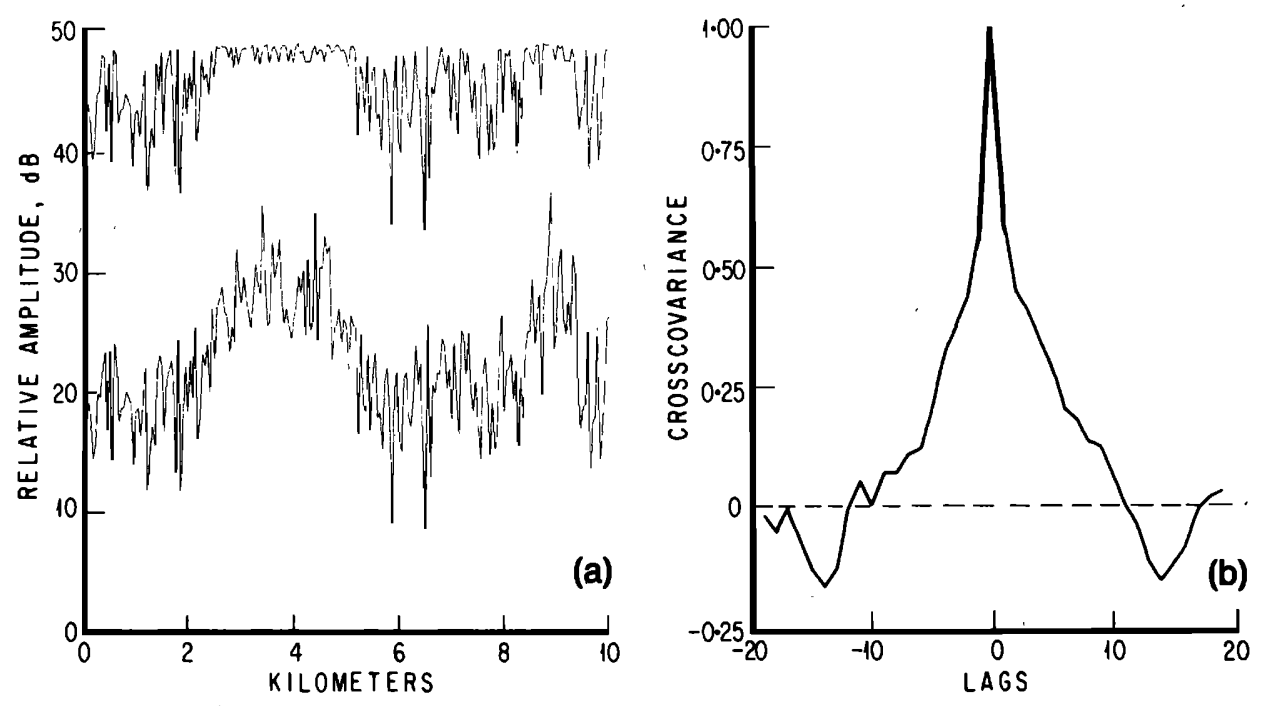

FIG. 5. Variations in the amplitude of the near-specular return which is clipped in the recorded data [ (a), top] are recovered from the corresponding mean sidelobe response [ (a), bottom]. Each data point corresponds to one ping. The cross covariance between these two time series, excluding the clipped portions, is shown in (b), where successive lags are successive pings. The mean sidelobe response was found to be $25 \mathrm{~dB}$ below the near-specular return. 
tablish a common reference between pings, the 16 beams are roll compensated and put into a set of beams $22 \mathrm{deg}$ apart, one of which is centered on the true vertical. Each roll-compensated beam is obtained by linear interpolation between the two adjacent unstabilized beams. Ideally, a ray-bending correction should also be applied. However, errors resulting from the omission of this correction are small at the steep angles of incidence considered here and are inconsequential in the scope of this paper. Again, roll-compensated beams for which sidelobe response and bottom return overlap are disregarded. The total energy in a return is then computed as the difference between the mean-square amplitude (signal + noise) in a predetermined window and the mean-square noise in an equivalent window. This way a fixed window size can be used for all beams.

As no accurate calibration exists for the Sea Beam system from which these acoustic data were recorded, the following results are given in relative units.

\section{RESULTS AND DISCUSSION}

Given that variations in bottom type and bottom roughness are the main factors in the nature of the fluctuations of the acoustic backscatter from the seafloor, data from three geologically different areas have been analyzed to determine what could be learned from the acoustics. Although it is
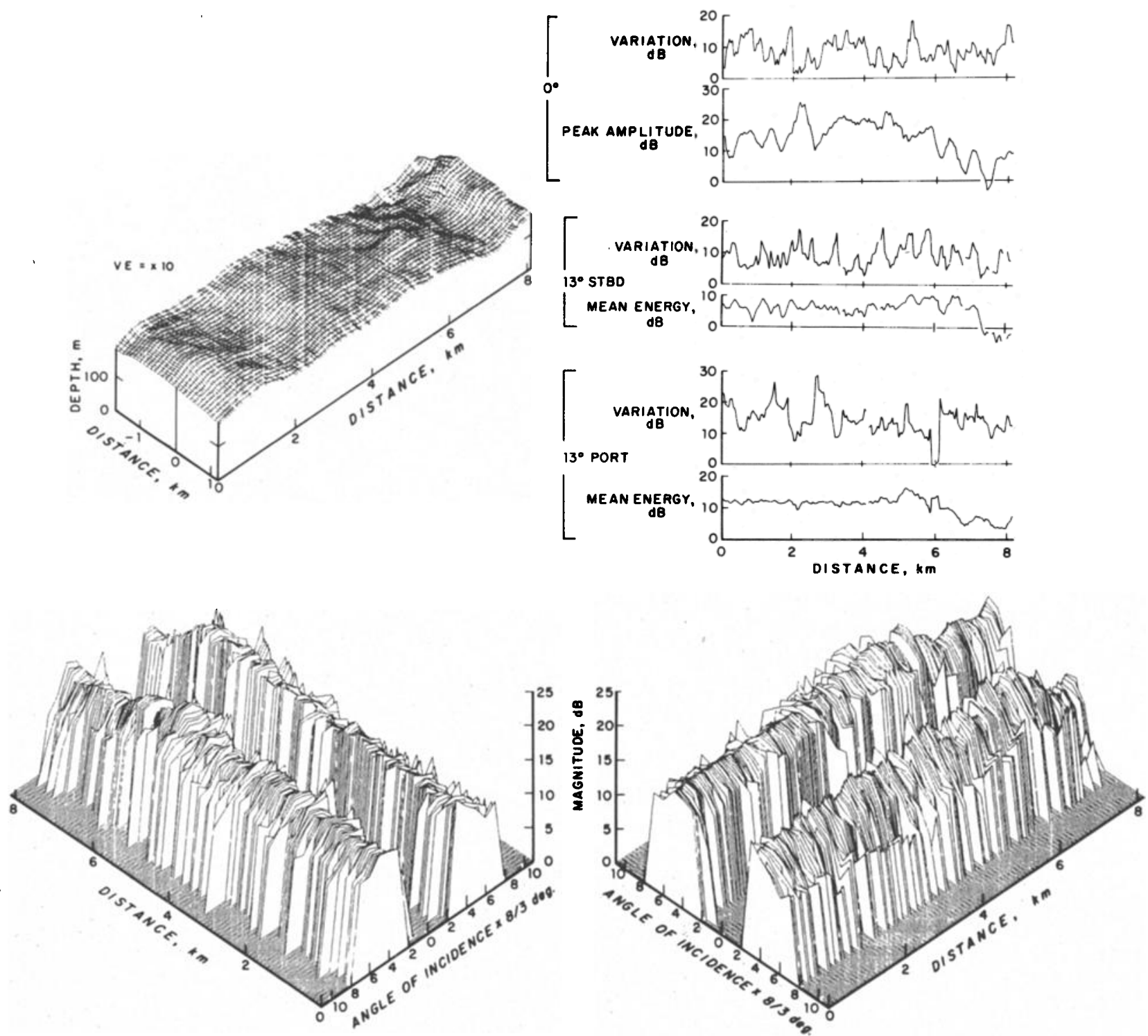

FIG. 6. Manganese nodule area: synopsis of Sea Beam data. Upper left: bathymetry for one swath of Sea Beam. The depth scale in meters is shown with a vertical exaggeration of 10 and its origin is at $4600 \mathrm{~m}$. The distance across and along track are in kilometers. Across track, a vertical bar indicates the position of normal incidence. The terrain has a very gentle undulation along track. Upper right: along-track variations of peak amplitude in the near-specular direction ( $\sim 0 \mathrm{deg}$ incidence) and of total energy at about 13-deg incidence. Plots have been low-pass filtered by averaging over five pings. The coefficient of variation (standard deviation/mean) shows the variability in the data for each of the three angles of incidence. Lower: partial angular dependence of total energy and its variations along track are displayed in both left and right view. The center portion (between $\pm 5 \frac{1}{3}$ deg incidence) has been left out because of sidelobe interference and saturation in the data. The notch seen on the outer starboard side is probably a system-related artifact, for it is found to some extent in all the recorded data. 

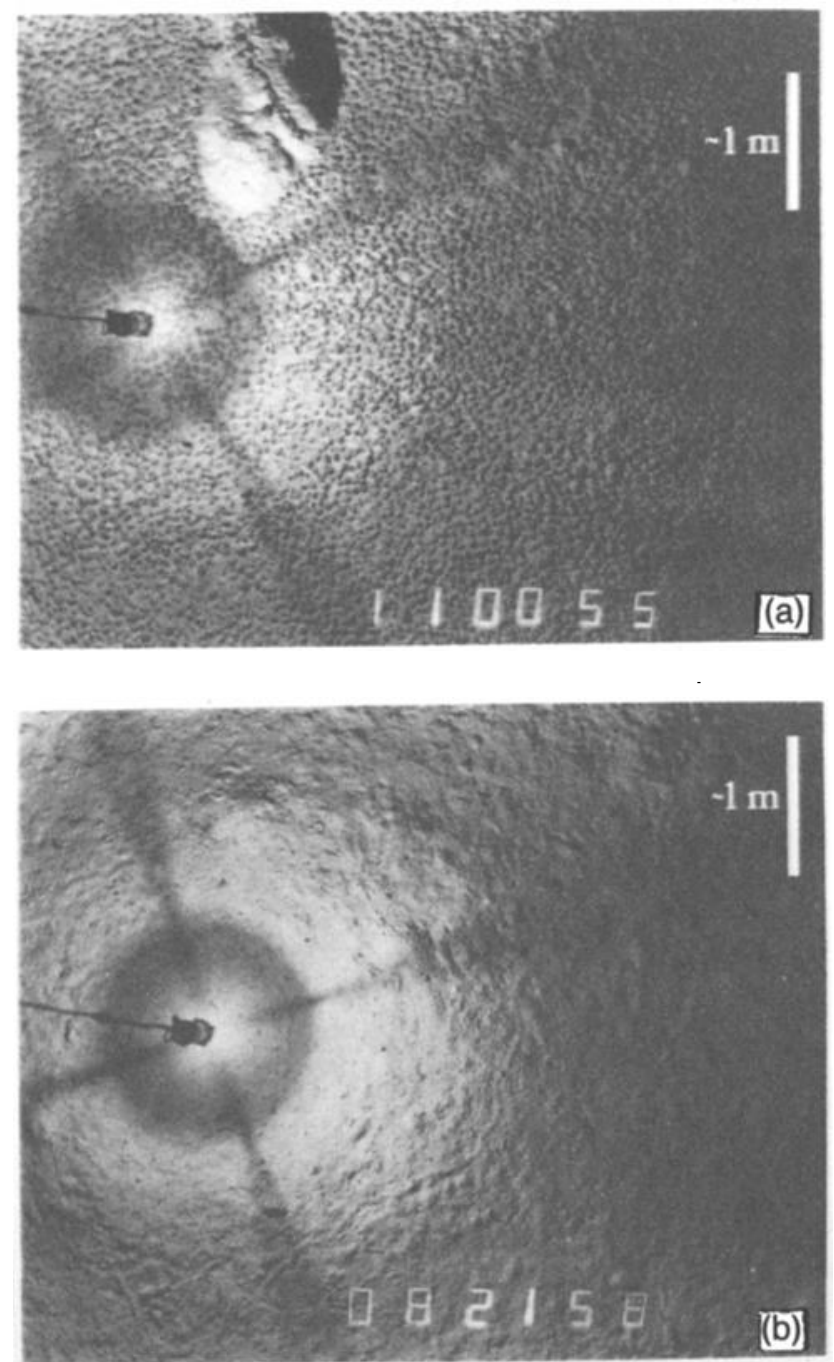

FIG. 7. Representative Deep Tow bottom photographs taken in the manganese nodule field. They show the contrast between a relatively dense coverage of manganese nodules (a) and bare mud (b).

possible to tell these areas apart from analysis of the acoustic data alone, our acoustic data base is too small and incomplete to identify them, and we use ground truth from independent measurements to validate the acoustic measurements made with Sea Beam.

The three areas investigated are: (1) a manganese nodule field in the North Eastern Tropical Pacific $\left(15^{\circ} \mathrm{N}\right.$, $125^{\circ} \mathrm{W}$ ) for which there are bottom photographs taken with MPL's Deep Tow instrument package, ${ }^{24}$ as well as box core data; (2) a sedimentary environment in the North San Clemente basin ( $\sim 150 \mathrm{~km}$ southwest of San Diego, CA) with Deep Tow bottom photographs in the general area; and (3) a lava sheet flow on the crest of the East Pacific Rise around $10^{\circ} \mathrm{N}$ with supporting data from the Lamont-Doherty Geological Observatory Sea MARC I sidelooking sonar ${ }^{25}$ and bottom photographs.

For each area, a composite figure (Figs. 6, 8, and 10) has been assembled to give a synopsis of the Sea Beam data. Sea Beam bathymetry is shown in a three-dimensional view of a single swath, as a mesh of instantaneous cross track depth profiles, low-pass filtered along track and displayed with a vertical exaggeration of 10 . The acoustic data are presented as along-track variations of relative peak amplitude in the specular direction and relative total energy for nonspecular beams. As described in Sec. II, the peak amplitude information has been recovered from sidelobe data, and the energy has been calculated only for nonspecular beams where bottom return and sidelobe response were well separated. In all displays, the data have been low-pass filtered with a running mean averaging over the number of pings necessary to traverse a single beam footprint $\left(2 \frac{2}{3} \mathrm{deg} \times 22_{3} \mathrm{deg}\right)$ on the seafloor at the depth considered. A coefficient of variation, which is simply the standard deviation normalized by the mean over the averaging interval, is also displayed to give a measure of the variability in the data. Finally, partial angular dependence profiles of total energy are stacked and lowpass filtered along track to show trends in the backscattered acoustic energy both along and across track. The central portion of the angular dependence of total energy has been purposely left out because of our inability to remove the sidelobe contribution contaminating the returns closest to the specular direction without seriously degrading the returns themselves.

In the following discussion, we assess how much can be learned about a portion of seafloor surveyed with Sea Beam by analysis of the acoustic backscatter it receives. We consider successively the system's ability to delineate acoustic boundaries, the use of an angular dependence function of backscattering to differentiate between various types of substrate, and the potential for estimating the microroughness of the bottom.

\section{A. Mapping acoustic boundaries}

Because most statistical analyses of the backscattered sound field are based upon the assumption of a homogeneous (stationary) scattering surface, it is important to be able to isolate seafloor areas for which this assumption holds. To this end, we define an acoustic boundary as the place where a marked change in trend appears in the acoustic data. With a multibeam system, such trends can be followed both along and across tracks, given that it is possible to correct for bottom slope in both directions. In this paper, we simplify the problem by limiting ourselves to nearly flat portions of seafloor.

\section{Manganese nodule area}

In a previous paper, ${ }^{26}$ we used the variations in amplitude of the specular beam alone to infer manganese nodule coverage over a well-documented nodule mining site. Although the estimates of coverage were crude, they were in very good qualitative agreement with estimates of coverage derived from Deep Tow bottom photographs of the same area. Our ability to correctly identify bare mud patches, areas sparsely covered with nodules, and areas densely covered throughout the mining field was a good indication that acoustic boundary mapping is feasible with Sea Beam. Here, we extend the analysis to nonspecular beams. 

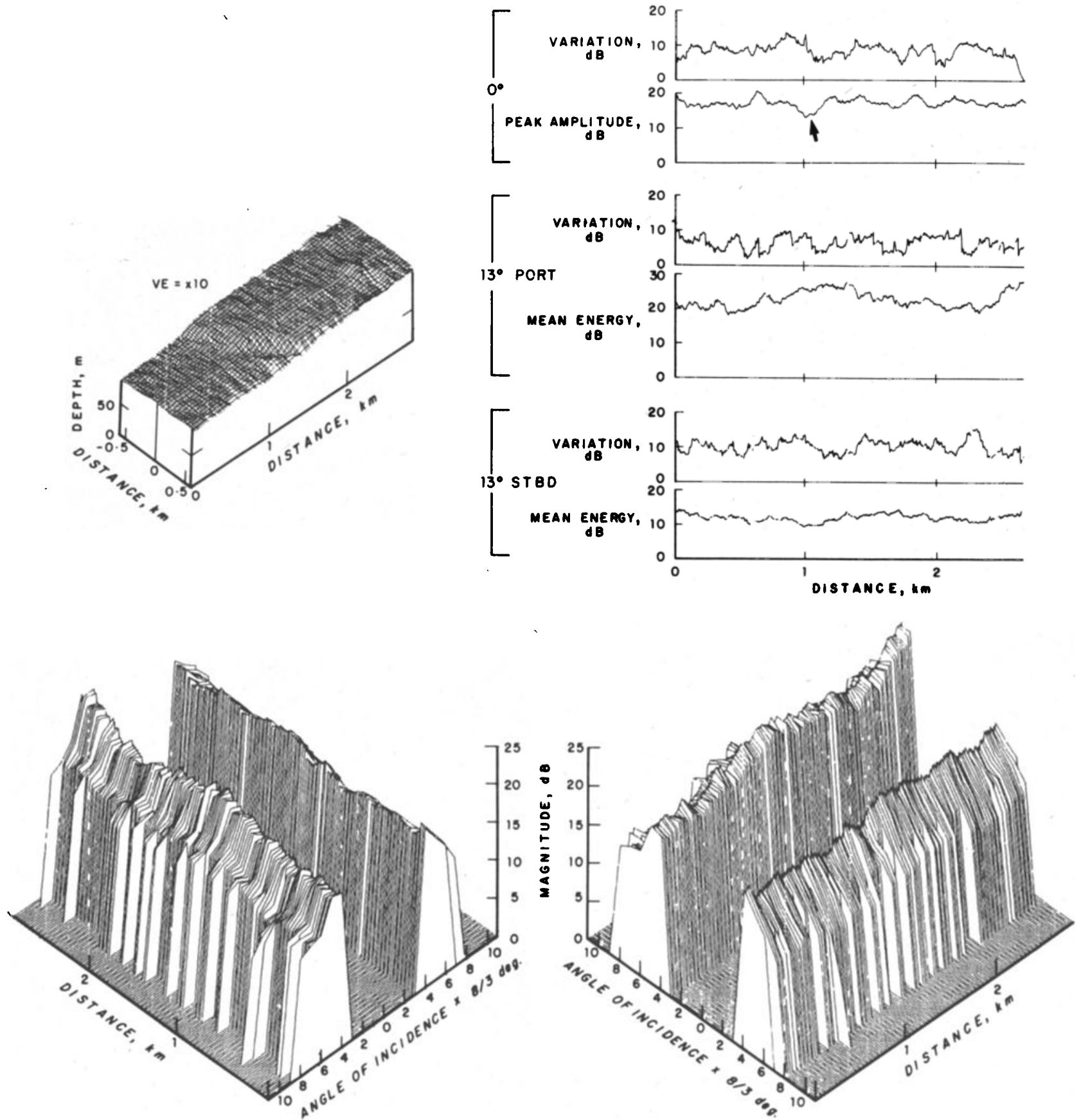

FIG. 8. North San Clemente Basin: synopsis of Sea Beam data. Layout and scales are the same as in Fig. 6. The origin of the depth scale is at $2000 \mathrm{~m}$. Depth is shown with a vertical exaggeration of 10 . A small step $(\sim 10 \mathrm{~m}$ high $)$ runs diagonal to the swath around the 1-km mark along track. Plots of amplitude and total energy data have been low-pass filtered by averaging over 22 pings. The small step in the bathymetry appears in the amplitude data (arrow) as a marked dip in amplitude. The gap in the center portion of the angular dependence of total energy is larger in this figure than in Fig. 6 because, as the water depth decreases, so does the time separation between arrivals on individual beams. As a result, the sidelobe interference affects more beams.

As shown in Fig. 6, the portion of seafloor considered is nearly flat, and the average depth is about $4500 \mathrm{~m}$. At this depth, the Sea Beam transmits every $8 \mathrm{~s}$ and, since the ship was moving at $\sim 5 \mathrm{~m} / \mathrm{s}(10 \mathrm{kn})$, the spatial sampling interval is roughly $40 \mathrm{~m}$ along track. By comparison, the diameter of the vertical incidence footprint is about $200 \mathrm{~m}$, so that it takes five pings to traverse a footprint. This number was used as the averaging interval in the low-pass filter of the data for the area.

With this averaging, the peak amplitude of normal inci- dence $(0 \mathrm{deg})$ returns is seen to fluctuate around a mean level of about $15 \mathrm{~dB}$ between 0 and $6 \mathrm{~km}$ and then drop sharply beyond the 6-km mark (Fig. 6). A similar trend, although much better defined, is seen in the mean total energy. For reference, \pm 13 -deg incidence corresponds to a distance across track of $\pm 1 \mathrm{~km}$ from the vertical incidence point. The drop in level is clearly seen in both port and starboard plots, but it happens $\sim 1 \mathrm{~km}$ further along track (7$\mathrm{km}$ mark) on the starboard side. It can be followed also on the stacked profiles of total energy, where it is seen to run 


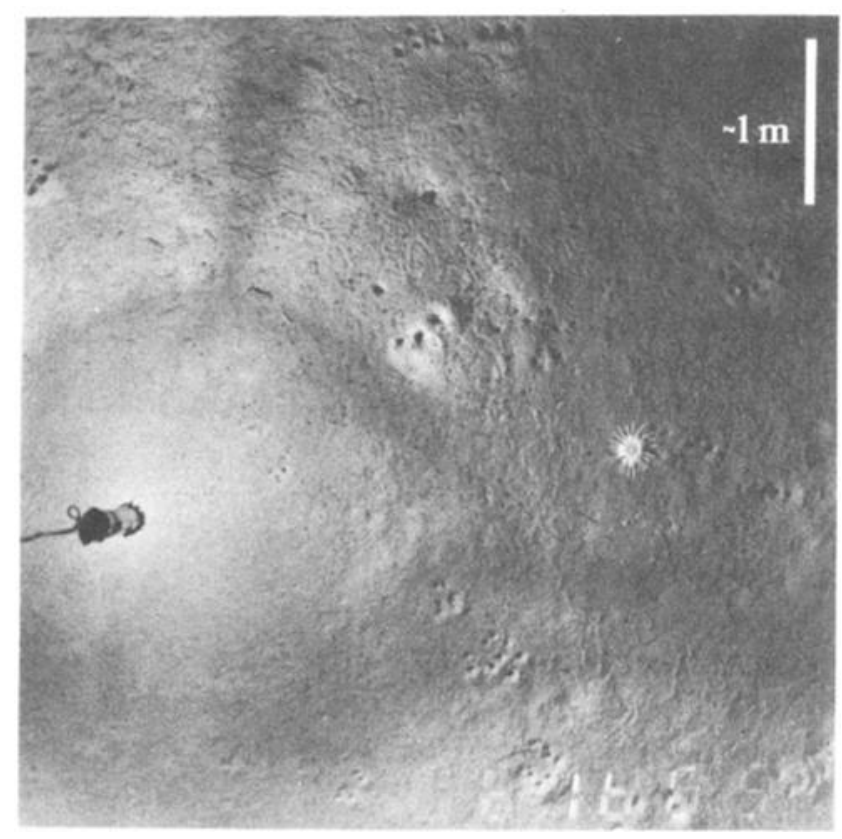

FIG. 9. Deep Tow bottom photograph taken in the North San Clemente basin. The sediments appear relatively smooth with occasional animal generated boreholes and craters, and numerous worm tracks.

diagonal to the ship's track. This diagonal therefore defines an acoustic boundary.

Deep Tow bottom photographs taken along the same track show that this boundary is associated with a rapid transition between dense nodule coverage [Fig. 7(a)] and bare mud [Fig. 7(b)]. In this case, the change in bottom substrate from nodules to mud, rather than their relative roughness, seems to be the dominant factor in the backscattering process. Also, it is interesting to note that, in this area, nonspecular beams energy is better suited for acoustic boundary mapping than peak amplitude in the specular return. The peak amplitude data shown in Fig. 6 seem to be more sensitive to small variations in bottom slope and to potential bottom focusing effects, and would therefore require more averaging to bring out the underlying trend readily observable in the energy data of nonspecular beams.

\section{North San Clemente basin}

The portion of Sea Beam data considered in Fig. 8 corresponds to a flat area around $32^{\circ} 30^{\prime} \mathrm{N}, 118^{\circ} 10^{\prime} \mathrm{W}$. The bathymetry shows a small diagonal step rising $10 \mathrm{~m}$ over $150 \mathrm{~m}$, and the average depth is $1900 \mathrm{~m}$. At this depth, Sea Beam transmits every $4 \mathrm{~s}$. In this instance, the ship speed was about $1 \mathrm{~m} / \mathrm{s}$ ( $\sim 2 \mathrm{kn}$ ), yielding a sampling interval along track of roughly $4 \mathrm{~m}$, so that 22 pings are necessary to transverse the length of a vertical incidence footprint $(88 \mathrm{~m})$. This number of pings was therefore used as the averaging interval to lowpass filter the data.

Because this averaging interval is large, the profile of normal incidence peak amplitude data appears relatively smooth, with less variability than that of Fig. 6. No major trend changes are observable in this profile (Fig. 8). The drop in amplitude (arrow) around the 1-km mark is most likely due to the small bathymetric step. The uniformity of this portion of seafloor is confirmed by the mean total energy data, even though a small undulation with a $1.5-\mathrm{km}$ wavelength is noticeable on both port and starboard. This trend is most marked at 13-deg incidence to port. Distances across track are $\mathbf{4 4 0}$ and $545 \mathrm{~m}$ from the vertical incidence point at 13-deg and 16-deg incidence, respectively. As seen in the stacked profiles of total energy, the undulations on port and starboard are not symmetric with respect to vertical incidence, but appear to be offset diagonally from each other in roughly the same orientation as the bathymetric step. A tenuous acoustic boundary can then be defined on this Sea Beam swath as a patch of higher backscatter extending 1.5 $\mathrm{km}$ along track and trending diagonally across track.

Owing to the complex nature of sedimentation patterns in this area, it is difficult to relate the patchiness observed in the acoustics to geological processes. Sediments of the San Clemente basin are of both turbidite and pelagic origin and contain mostly fine grained sand and muds (biogenic and/or micaceous) ${ }^{27,28}$ Deep Tow bottom photographs taken in the vicinity (Fig. 9) show a smooth sedimentary bottom with evidence of intense animal activity (worm tracks and feces, holes, and craters, etc.) so that bioturbation must play a major role in the vertical distribution of sediments. Several speculations can be made to account for the acoustic boundary observed. It could be due to a patch of seafloor where sand has been bioturbated with the overlying mud, thereby enhancing its backscattering properties. $A$ thinning of the surficial mud layer over sand would have a similar effect. Another possibility is a change in the fine-scale roughness of the bottom as a result of animal activity, higher backscattered energy corresponding to a rougher interface. More data (subbottom profiles and/or cores) are necessary to determine whether roughness or bottom type or a combination dominates the backscattering process in this area.

\section{Rise crest environment}

The acoustic data presented in Fig. 10 correspond to the section of bathymetry delimited by the arrows. In those bounds, the seafloor is nearly flat, with an average depth of $2560 \mathrm{~m}$. With a 6-s ping rate and a ship speed of about $1 \mathrm{~m} / \mathrm{s}$, the along-track sampling interval is $6 \mathrm{~m}$ (compared to a footprint $120 \mathrm{~m}$ in diameter), and the averaging interval used for low-pass filtering of the data is 20 pings.

The same smoothing effect of large averaging intervals noted in the San Clemente basin data is seen here. However, peak amplitude variations are relatively higher indicating a greater variability in the raw data. Small trend changes over along-track distances $1 \mathrm{~km}$ or less are noticeable in the peak amplitude as well as in the total energy data. They can also be followed in the stacked profiles of total energy, which show no along-track symmetry about vertical incidence. In this case, relation of the acoustic data to the geological processes is facilitated by the uniqueness of the area and the availability of independent measurements made simultaneously with the Sea MARC I system.

This region of the East Pacific Rise around $10^{\circ} 05^{\prime} \mathrm{N}$ is characterized by nearly flat-lying basalt sheet flows covering an area over $10 \mathrm{~km}$ long and $3 \mathrm{~km}$ wide on the rise axis. ${ }^{29} \mathrm{~A} 5$ $\mathrm{km}$ segment from this area imaged with the Sea MARC I 

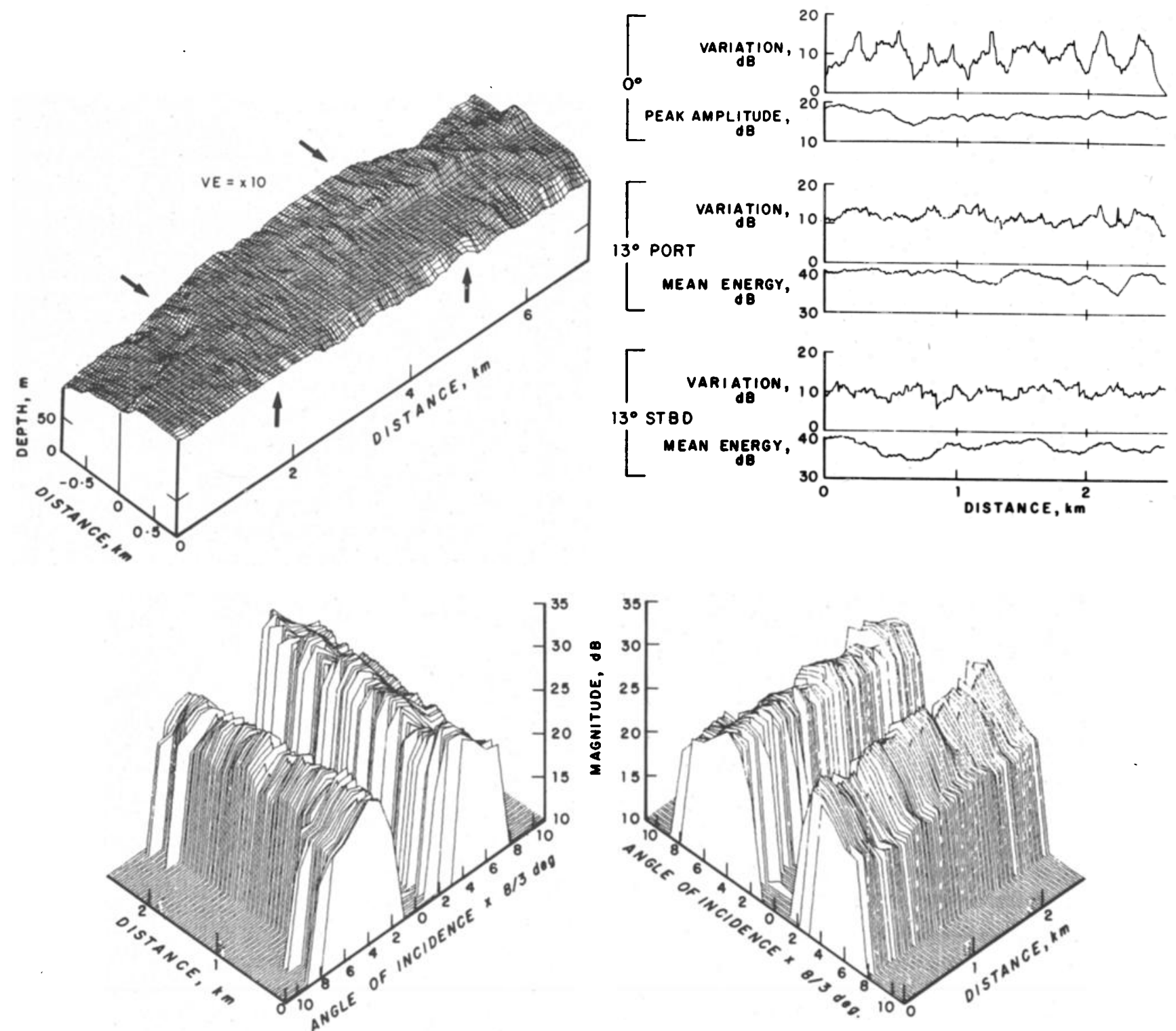

FIG. 10. Lava sheet flow on the crest of the East Pacific Rise: synopsis of Sea Beam data. Layout and scales are identical to those of Figs. 6 and 8 . The origin of the depth scale is at $2700 \mathrm{~m}$. The acoustic data displayed correspond to the section of bathymetry $(\sim 2.7 \mathrm{~km} \mathrm{long})$ delimited by the arrows. In this section, the bathymetry is nearly flat. For compactness of this figure, the origin (0) of the vertical axes for the acoustic data is not shown; however, the scale is the same as in Figs. 6 and 8.

side-looking sonar system is shown in Fig. 11(a). On this image, acoustic shadows are white and intense backscatter is black. A line drawing [Fig. 11(b)] of this image indicates fissures and flow channels observable in the sidescan data as well as in the area covered by the Sea Beam acoustic data considered here (dotted line). The basalts are fresh as evidenced by their glass coatings and the paucity of sediment seen in bottom photographs in the area [Fig. 11(c)]. Patches of benthic organisms seen in the bottom photographs and temperature measurements also indicate that this region of the rise axis is hydrothermally active. ${ }^{29}$

The distribution of flow channels outlined inside the dotted line [Fig. 11(b)] coincides remarkably well with the pattern seen in the total-energy profiles (Fig. 10) at $\pm 13-$ deg incidence ( $\sim 590 \mathrm{~m}$ across track from the point of vertical incidence). Total energy is high between flow channel zones and drops upon crossing the zones. These flow channels appear in the sidescan image [Fig. 11(a)] as a lighter shade of grey than their surroundings, indicating reduced backscattering properties. Because this portion of seafloor is uniform in type (basalts) and nearly flat, the change in backscattering properties is most likely due to a change in the microroughness. We therefore conclude that in this area the acoustic backscatter is dominated by bottom microroughness.

\section{B. Angular dependence}

Having isolated acoustically homogeneous areas of the seafloor by defining acoustic boundaries, one would like to use the acoustic data to identify the type of bottom within each area. Because Sea Beam measures acoustic backscatter simultaneously at 16 angles of incidence spaced $2 \frac{2}{3}$ deg apart 

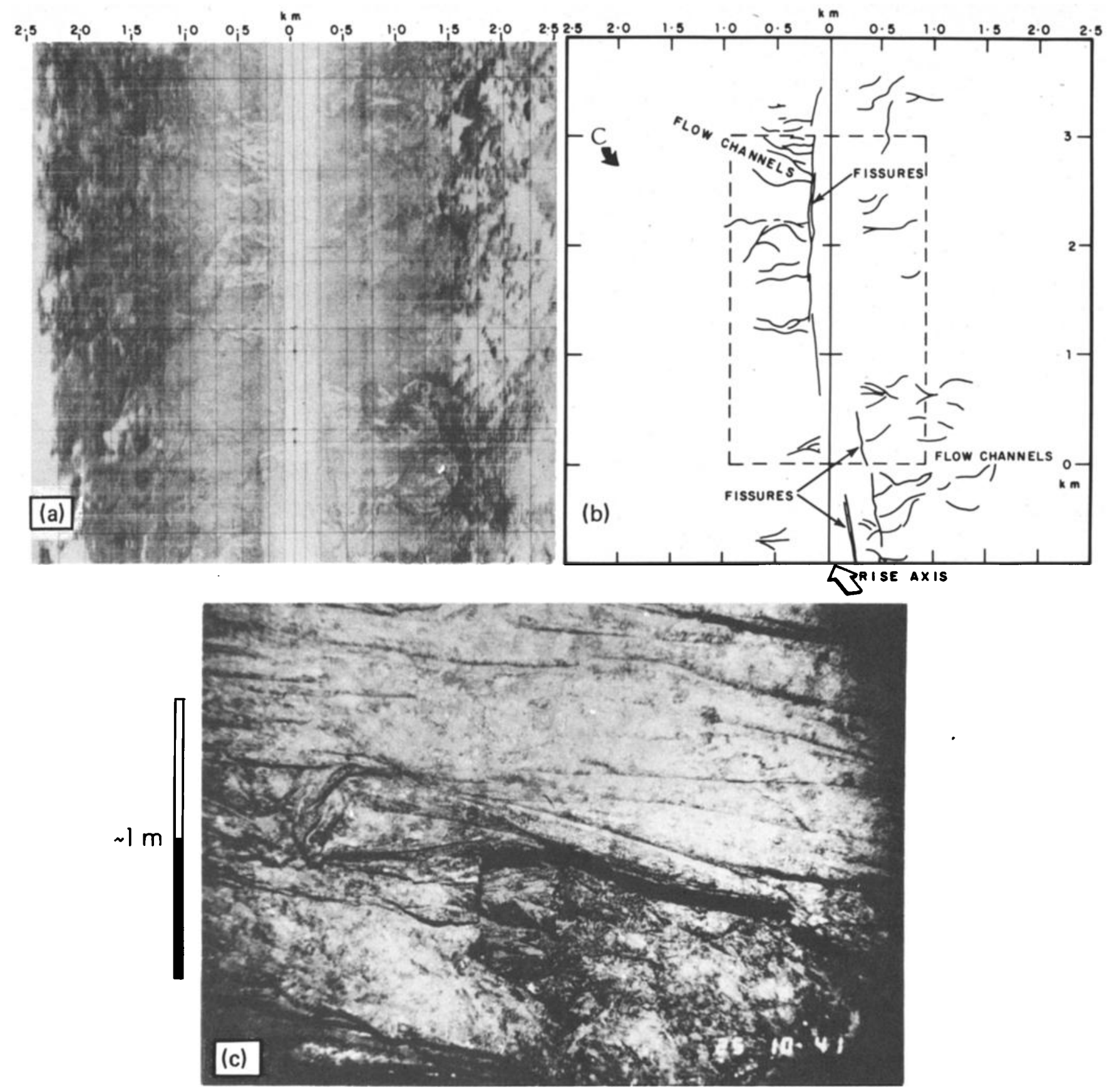

FIG. 11. Sea MARC I side looking sonar image of the lava sheet flow (a). The swath covered by Sea Beam is delimited by the dotted line. On this image, acoustic shadows are white, and intense backscatter is black. Fissures and flow channels observable in this image are outlined in (b) where distance along track originates at the same location as the $0-\mathrm{km}$ point of the acoustic data in Fig. 10. The arrow points to the location of the bottom photograph (c).

(usually over $\pm 20 \mathrm{deg}$ from vertical when the ship is not rolling), a discrete angular-dependence function is readily obtainable. This function is a potential criterion for differentiating between bottom types.

As mentioned previously, it has not been possible to obtain the complete angular dependence from the data presented here because of saturation and sidelobe interference. As a result, only the tails of the function are shown in Fig. 12 for the three types of seafloor considered. In this figure, the levels indicated correspond to the relative total energy measured at each angle over flat, acoustically homogeneous regions and corrected for transmission loss.
The tails of these three angular-dependence functions are mostly remarkable for the differences in their relative energy levels. Hemipelagic sediments (sand and mud) appear to be about $10 \mathrm{~dB}$ above nodules and $10 \mathrm{~dB}$ below basalts. Although very few deep sea data in the kilohertz range exist in the literature, these relative levels are in general agreement with comparable measurements in coastal locations summarized by Urick. ${ }^{30}$ In spite of the very different nature of the three areas considered, the shape of their partial angular-dependence function is similar (Fig. 12), and, therefore, cannot be used to identify them. Consequently, in our data, the overall difference in levels between the bottom 


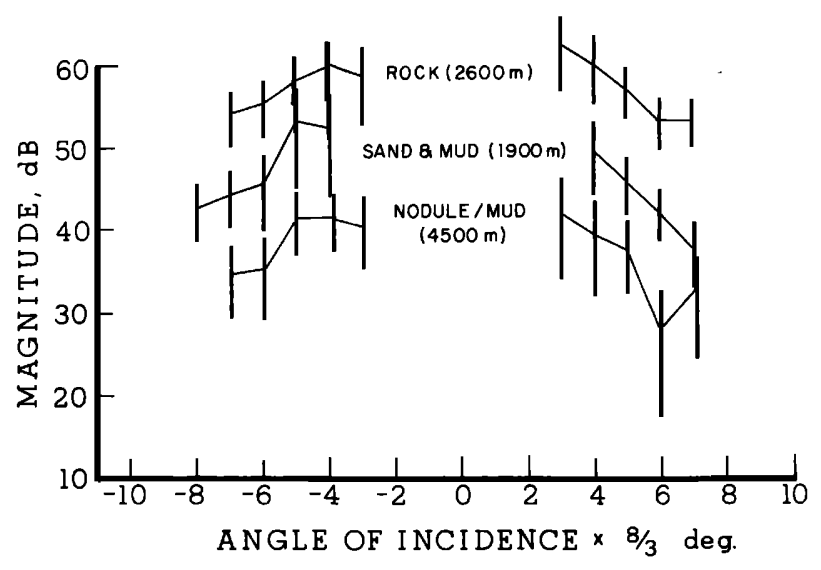

FIG. 12. Angular dependence of total backscattered energy. The tails of the angular dependence function shown here for the three types of seafloor considered are remarkable for the differences in their energy level. However, their shapes are too similar to tell them apart on that criterion alone. Vertical bars represent $1 \mathrm{~s}$. d. about the mean value. The label "nodule/mud" corresponds to dense nodule coverage over a mud substrate; "sand and mud" indicates hemipelagic sediments found in the North San Clemente basin; and "rock" refers to basalts from the lava sheet flow on the East Pacific Rise at $10^{\circ} \mathrm{N}$.

types is the only tangible criterion available on which to separate them. However, we are missing the information contained in the beams near normal incidence, which, when expressed as the ratio of the specular backscattered energy to that of the adjacent nonspecular beams could prove a good indicator of the nature of the bottom.

To confirm this, a modification of the MPL Sea Beam acoustic data acquisition system is in progress to record both amplitude and phase of the backscattered signals. Sidelobe interference can then be removed without degrading the bottom return to produce a complete angular dependence function.

\section{Estimates of surface statistics}

In the present data set, we are restricted to echo envelope statistics for estimating parameters of the rough surface such as rms roughness and correlation area. For small to moderate relative roughness $\left(4 \kappa^{2} \sigma^{2}<1\right)$, these parameters are directly related to the shape of the pdf of normal-incidence echo envelopes through the ratio $\gamma$ of coherent to incoherent energy in the echo [Eqs. (9) and (10) ]. Estimates of $\gamma$ are obtained by fitting a Rician distribution [Eq. (11)] to the histogram of normal incidence echo peak amplitude. This method has the advantage of being independent of system calibration. Therefore, peak-amplitude variations recovered from sidelobe levels (e.g., Fig. 5) can be used to produce a histogram. Three such histograms for the three areas under consideration are shown in Fig. 13. The overlying curves represent the "best" fit, in a chi-square goodness of $\mathrm{fit}^{31}$ sense, of a Rician distribution to the corresponding values of $\gamma$. The fit is not good in all three cases and a different distribution (e.g., extremal pdf) ${ }^{32}$ may yield a better fit; however, the Rician distribution is the only one which relates to physical parameters. It is used here for this reason. The three areas yield markedly different values of $\gamma$, indicat-

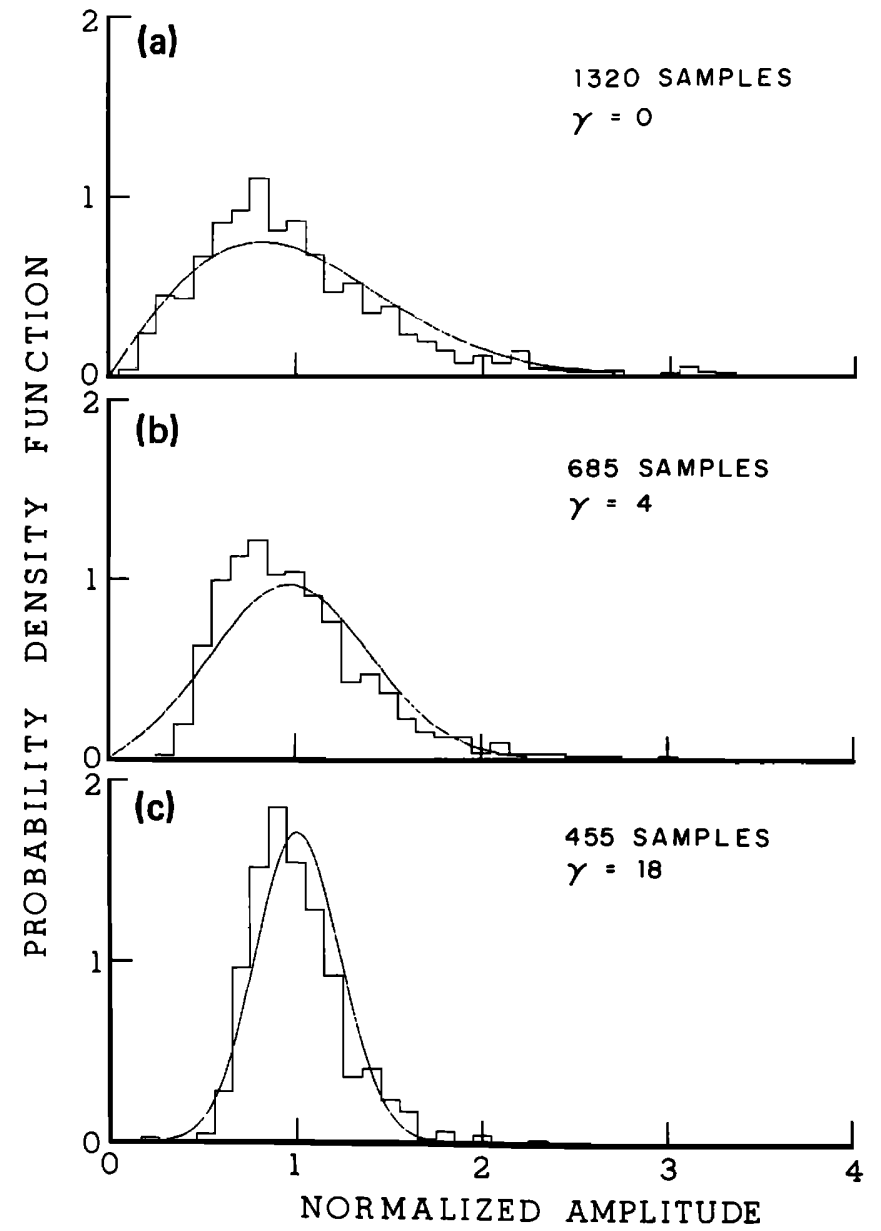

FIG. 13. Probability density functions of peak amplitude in the near-specular returns for the three types of seafloor. (a) Manganese nodule area; (b) sediments in the North San Clemente basin; and (c) lava sheet flow on the crest of the East Pacific Rise. Histograms are from Sea Beam data. Superimposed curves represent the corresponding "best" fit of a Rician pdf with the parameter $\gamma$ holding the values indicated in each case.

ing differences in their roughness structure. We can estimate the rms roughness of each area from the bottom photographs and calculate an approximate correlation area by using Eq. (9) and the values of $\gamma$ given in Fig. 13. With Sea Beam, $\kappa=2 \pi / \lambda \simeq 51$ and $\Delta \phi=\Delta \chi=2 \frac{2}{3}$ deg so that at normal incidence $\alpha=\beta \simeq 2.8$ in Eq. (9).

In the manganese nodule area, $\gamma=0$, indicating an infinitely rough surface according to Eq. (9). In fact, the rms roughness estimated from box cores and bottom photographs is about $2 \mathrm{~cm},{ }^{26}$ and $4 \kappa^{2} \sigma^{2}=4.15>1$ so that the assumptions leading to Eq. (9) are violated. The surface can therefore be considered rough for our purposes. By comparison, $\gamma=4$ for the North San Clemente basin data and $\gamma=18$ for the basalt sheet flow, indicating relatively smoother surfaces. With these two values of $\gamma$, Eq. (23) yields $\sigma^{2} l_{1} l_{2}=2.0310^{-5} \mathrm{~m}^{4}$ and $4.510^{-6} \mathrm{~m}^{4}$, respectively. If $\sigma$ were the same for both regions, the correlation areas $\left(l_{1} l_{2}\right)$ would differ by a factor of 5 , making it possible to tell the two types of bottom apart. Using the roughness $(2 \mathrm{~cm})$ in the nodule area as a photographic reference scale, it is reasonable to assume $\sigma<1 \mathrm{~cm}$ for the two other regions. This yields a lower bound on the correlation area of $0.203 \mathrm{~m}^{2}$ in the San Clemente basin and $0.045 \mathrm{~m}^{2}$ on the basalt sheet flow. 
Both correlation areas support the assumptions which lead to Eq. (9). In the San Clemente basin, the Gaussian assumption is justified because the surface microroughness is due mostly to animal activity and is therefore randomly distributed with many irregularities within the ensonified area. These irregularities are most likely isotropic so that $l_{1} \simeq l_{2} \simeq 45 \mathrm{~cm}$. In the rise-crest data, the irregularities are presumably anisotropic. By analogy with current generated ripples, roughness in the direction perpèndicular to the flow of lava probably has a longer correlation length than roughness parallel to the flow which is typically characterized by linear wrinkles [Fig. 11(c)]. From bottom photographs taken in the general area, the spacing between wrinkles appears random, giving some justification for the Gaussian assumption. The limiting values required to satisfy $\alpha l_{1}^{2}+\beta l_{2}^{2}=1$ in Eq. (7) given that $l_{1} l_{2}=0.045 \mathrm{~m}^{2}$ are $l_{1} \simeq 60 \mathrm{~cm}$ and $l_{2} \simeq 7.5 \mathrm{~cm}$. Although the value of the correlation area $\left(l_{1} l_{2}\right)$ is based on an empirical estimate of the rms roughness $\sigma$, the values obtained for $l_{1}$ and $l_{2}$ are consistent with the general roughness character observed in the area. It follows that in the limit $4 k^{2} \sigma^{2}<1$ (rms roughness of the order of $1 \mathrm{~cm}$ or less at $12 \mathrm{kHz}$ ), two seafloor environments with the same rms roughness can be differentiated by their correlation area.

\section{CONCLUSIONS}

Deep seafloor acoustic backscatter data measured with a Sea Beam system have been shown to hold a wealth of information on the nature of the seafloor surveyed. Acoustic boundaries are mapped over flat areas by following trend changes in acoustic data (peak amplitude at normal incidence and total energy otherwise) both along and across track. Clues to the nature of the bottom are found in the overall energy level of a partial angular-dependence function of backscattering as well as in the shape of the pdf of normalincidence echo envelopes, which is related to the degree of coherence in the backscattered acoustic field.

Data from three geologically different environments (a manganese nodule field, a hemipelagic sedimentary basin, and a rise-crest basalt sheet flow) have been analyzed and validated with independent measurements (bottom photographs and side-looking sonar data). The backscattering process seemed to be more sensitive to bottom type in the manganese nodule area, to bottom roughness in the risecrest data, and to a combination of both in the sedimentary basin. Total energy in the partial angular-dependence function was highest for basalts on the rise crest; it was roughly $10 \mathrm{~dB}$ lower for hemipelagic sediment in the San Clemente basin and another $10 \mathrm{~dB}$ lower for manganese nodules.

The shape of the pdf of echo envelopes was indicative of a rough surface in the manganese nodule area, and of smoother surfaces in the two other environments. This was confirmed by bottom photographs. When given the same roughness, the sedimentary basin and the rise-crest sheet flow were found to have quite different correlation areas. For small roughness $\left(4 k^{2} \sigma^{2}<1\right)$ the correlation area is therefore a useful parameter to differentiate between seafloor types having the same roughness.

\section{ACKNOWLEDGMENTS}

Funds for this research were provided by the Office of Naval Research (Contract No. N00014-79-C-0472). Data collection at sea was made possible through the cooperative efforts of the officers, crew, and scientific parties of $R / V$ ThOMAS WASHINGTON during expeditions CERES-leg 1 and PASCUA leg 1 and 5. The author wishes to thank $K$. Crane for making Sea MARC I data available and providing helpful comments, V. C. Anderson, F. N. Spiess, C. W. Helstrom, E. L. Winterer, and D. Alexandrou for valuable suggestions, R. C. Tyce for initiating the Sea Beam acoustic backscatter experiment at MPL, and F. V. Pavlicek for his support during the devlopment of the MPL Sea Beam acoustic data acquisition system. He is also grateful to E. Ford for typing and editing and J. Griffith for the art work.

\section{APPENDIX A}

From Eq. (1) in the text, the mean-square pressure is

$$
\left\langle p^{2}\right\rangle \approx B_{1}^{2} \iiint_{-\infty}^{\infty} \int D_{1}\left\langle\exp \left[2 j c\left(\zeta-\zeta^{\prime}\right)\right]\right\rangle d x d y d x^{\prime} d y^{\prime},
$$

with

$$
\begin{aligned}
D_{1}= & D_{0} D_{0}^{\prime} \exp 2 j\left\{a\left(x-x^{\prime}\right)+\kappa / 2 R_{0}\right. \\
& \left.\times\left[\left(x^{2}-x^{\prime 2}\right) \cos ^{2} \theta+y^{2}-y^{\prime 2}\right]\right\} .
\end{aligned}
$$

The average within the integral is the joint characteristic function of $\xi$ and $\xi^{\prime}$. Similarly,

$$
\begin{aligned}
\langle p\rangle^{2} & =\langle p\rangle\left\langle p^{*}\right\rangle \\
& =B_{1}^{2} \int_{-\infty}^{+\infty} \int_{-\infty}^{\infty} D_{1}(\exp 2 j c \zeta\rangle\left\langle\exp \left(-2 j c \zeta^{\prime}\right)\right\rangle d x d y d x^{\prime} d y^{\prime}
\end{aligned}
$$

so that the mean-square second moment of $p$ is

$$
\begin{aligned}
\left\langle S^{2}\right\rangle \approx B_{1}^{2} \iint_{-\infty}^{+\infty} \int_{-\infty} D_{1}\left[\left\langle\exp 2 j c\left(\xi-\zeta^{\prime}\right)\right\rangle\right. \\
\left.-\langle\exp 2 j c \xi\rangle\left\langle\exp \left(-2 j c \xi^{\prime}\right)\right\rangle\right] d x d y d x^{\prime} d y^{\prime}
\end{aligned}
$$

Using a Gaussian illumination function for $D_{0}$,

$$
D_{0}=\exp \left(-x^{2} / X^{2}-y^{2} / Y^{2}\right),
$$

where $X$ and $Y$ are the semiminor (respectively major) axes of the ellipse outlined by the intersection of the mainlobe of the beam pattern and the scattering surface.

For beamwidths $\Delta \mathcal{X}$ and $\Delta \phi$ in the $x$ and $y$ directions, respectively, we have

$$
X=R \sin \Delta \chi / \cos \theta, \quad Y=R \sin \Delta \phi,
$$

where $\theta$ is the angle of incidence of the beam. We assume spatial stationarity for the surface which means that the joint characteristic function of $\zeta$ and $\zeta^{\prime}$ depends only on the distance between points on the surface through $\xi=x-x^{\prime}$ and $\eta=y-y^{\prime}$, and that the correlation function depends only on $\xi$ and $\eta: C=C(\xi, \eta)$. Then, with the change of variables, ${ }^{5,6}$ 


$$
\begin{array}{ll}
x=x^{\prime \prime}+\xi / 2, & x^{\prime}=x^{\prime \prime}-\xi / 2, \\
y=y^{\prime \prime}+\eta / 2, & y^{\prime}=y^{\prime \prime}-\eta / 2,
\end{array}
$$

substituting (A6) and (A4) into (A3) and integrating over $x^{\prime \prime}$ and $y^{\prime \prime}$ yields

$$
\begin{aligned}
\left\langle S^{2}\right\rangle= & B_{2} \int_{-\infty}^{+\infty} \int_{2}^{\infty}\left[\left\langle\exp 2 j c\left(\xi-\zeta^{\prime}\right)\right\rangle\right. \\
& \left.-\langle\exp 2 j c \xi\rangle\left\langle\exp \left(-2 j c \xi^{\prime}\right)\right\rangle\right] d \xi d \eta
\end{aligned}
$$

with

$B_{2}=\frac{B_{1}^{2} \pi X Y}{2}=\frac{B^{2} R^{2} k^{2} X Y}{8 \pi R_{0}^{2} \cos ^{2} \theta}$

$D_{2}=\exp \left[-\left(2 a j \xi+\alpha \xi^{2}+\beta \eta^{2}\right)\right]$,

$\alpha=\frac{1}{2}\left(\frac{X^{2} \kappa^{2} \cos ^{4} \theta}{R_{0}^{2}}+\frac{1}{X^{2}}\right), \quad \beta=\frac{1}{2}\left(Y^{2} \frac{\kappa^{2}}{R_{0}^{2}}+\frac{1}{Y^{2}}\right)$.

Under the assumption of a normally distributed surface roughness, the characteristic and joint characteristic functions become $\mathrm{e}^{33}$

$\langle\exp 2 j c \zeta\rangle=\exp \left(-2 c^{2} \sigma^{2}\right)$,

$\left\langle\exp 2 j c\left(\xi-\xi^{\prime}\right)\right\rangle=\exp \left\{-4 c^{2} \sigma^{2}[1-C(\xi, \eta)]\right\}$,

where $c^{2}=\kappa^{2} \cos ^{2} \theta$, so that

$$
\langle p\rangle^{2}=\left(B_{2} \pi / \sqrt{\alpha \beta}\right) \exp \left(-4 c^{2} \sigma^{2}-a^{2} / \alpha\right),
$$

and

$$
\left\langle S^{2}\right\rangle=B_{2} \int_{-\infty}^{+\infty} \int_{-\infty}^{\infty}\left\{\exp \left[4 c^{2} \sigma^{2} C(\xi, \eta)\right]-1\right\} d \xi d \eta .
$$

Hence, we get the result

$$
\begin{aligned}
\gamma^{-1}= & \left\langle S^{2}\right\rangle /\langle p\rangle^{2} \\
= & \frac{\sqrt{\alpha \beta}}{\pi} \exp \left(\frac{a^{2}}{\alpha}\right) \int_{-\infty}^{+\infty}\left[\exp -\left(2 a j \xi+\alpha \xi^{2}+\beta \eta^{2}\right)\right] \\
& \times\left\{\exp \left[4 c^{2} \sigma^{2} C(\xi, \eta)\right]-1\right\} d \xi d \eta
\end{aligned}
$$

which relates the degree of coherence of the backscattered field to the parameters of the rough surface $\sigma$ and $C$.

This result is general in the sense that no limitations have been imposed on the wavelength of the sound radiation or equivalently on the length scale of the surface roughness. We have only assumed that the surface satisfies the Kirchhoff boundary condition, which requires there be no sharp edges on the scattering surface. We have also assumed that the roughness is normally distributed and that it is spatially stationary by casting the correlation function $C$ as a function of the distance between points on the surface $[C(\xi, \eta)]$. Although the applicability of such statistical properties to the ocean floor may seem questionable, especially with regard to stationarity, they are useful in reducing the foregoing integrals to more manageable expressions.

Outside of normal incidence, the coherent component of backscattering is likely to be small or negligible compared to the incoherent component, so that $\gamma$ will tend to zero. Nor- mal incidence is more interesting in that both components are then equally likely to dominate, depending on the type of rough surface, and there is a direct relationship between $\gamma$ and the shape of the pdf of normal incidence echo envelopes.

\section{APPENDIX B: ENVELOPE DISTRIBUTIONS}

For surfaces rough compared to an acoustic wavelength, the phase of the backscattered echo is, to a good approximation, distributed uniformly over the interval $(0,2 \pi)$, and the set of pressures received can be assumed to be normally distributed with variance $\sigma_{p}^{2}$. From the central limit theorem, this approximation is better as the set gets larger ( $\gtrsim 30$ ). Under such conditions, the amplitude $E$ of the echo has been shown ${ }^{10,16}$ to be Rayleigh distributed with a pdf:

$$
W(E)=\left(E / \sigma_{p}^{2}\right) \exp \left(-E^{2} / 2 \sigma_{p}^{2}\right) .
$$

The various moments of $E$ are, by definition,

$$
\left\langle E^{n}\right\rangle=\int_{-\infty}^{+\infty} E^{n} W(E) d E,
$$

from which, after substitution of Eq. (B1), we obtain the second moment,

$$
\left\langle E^{2}\right\rangle=2 \sigma_{p}^{2} .
$$

The Rayleigh pdf can then be expressed in terms of the amplitude of the echo envelope $E$ and its mean-square value $\left\langle E^{2}\right\rangle$, two quantities readily measurable in our data:

$$
W(E)=\left(2 E /\left\langle E^{2}\right\rangle\right) \exp \left(-E^{2} /\left\langle E^{2}\right\rangle\right) .
$$

The generalized Rayleigh distribution or Rice distribution ${ }^{19}$ has been used also to describe the effects of coherent scattering on the pdf of the echo envelope. ${ }^{10,12}$ In this case, the backscattered pressure wave is considered as the sum of a coherent component of rms value $A$ and an incoherent component normally distributed with variance $\sigma_{p}^{2}$, respectively analogous to the sine wave and the narrow-band Gaussian noise of the original Rician distribution.

The corresponding echo envelope $E$ is distributed with a pdf:

$$
W(E)= \begin{cases}\frac{E}{\sigma_{p}^{2}} \exp \left(-\frac{E+A^{2}}{2 \sigma_{p}^{2}}\right) I_{0}\left(\frac{E A}{\sigma_{p}^{2}}\right), & E \geqslant 0, \\ 0, & \text { otherwise }\end{cases}
$$

where $I_{0}$ is the zeroth order modified Bessel function. Remembering Eq. (B2), the moments of $E$ are given by

$$
\left\langle E^{n}\right\rangle=\left(2 \sigma_{p}^{2}\right)^{n / 2} \Gamma\left(\frac{n}{2}+1\right)_{1} F_{1}\left(-\frac{n}{2} ; 1,-\frac{A^{2}}{2 \sigma_{p}^{2}}\right) .
$$

For even-order moments, the confluent hypergeometric function ${ }_{1} F_{1}(-n ; 1, n r)$ is equal to the $n$th Laguerre polyno$\mathrm{mial}^{34}$

$$
L_{n}(r)=\sum_{i=1}^{n}(-r)^{i} \frac{n !}{(i !)^{2}(n-i) !} .
$$

Therefore, the second moment of $E$ is

$$
\left\langle E^{2}\right\rangle=2 \sigma_{p}^{2}+A^{2} .
$$

To express the Rician distribution in terms of readily measurable quantities, it is convenient to define a parameter 


$$
\gamma=A^{2} / \sigma_{p}^{2},
$$

which is the coherent to incoherent power ratio in the echo. Substitution of Eqs. (B8) and (B9) into (B5) yields

$$
\begin{aligned}
W(E)= & (2+\gamma) \frac{E}{\left\langle E^{2}\right\rangle} \exp \left(-\frac{(2+\gamma) E^{2}+\gamma\left\langle E^{2}\right\rangle}{2\left\langle E^{2}\right\rangle}\right) \\
& \times I_{0}\left\{\left(E /\left\langle E^{2}\right\rangle^{1 / 2}\right)[\gamma(2+\gamma)]^{1 / 2}\right\} . \quad(\mathrm{B} 10)
\end{aligned}
$$

When $\gamma<1$, Eq. (B10) tends to the Rayleigh distribution [Eq. (B4)]; conversely, when $\gamma>1$, Eq. (B10) tends to a Gaussian-like distribution:

$$
W(E) \simeq\left(\gamma X / 2 \pi\left\langle E^{2}\right\rangle\right)^{1 / 2} \exp \left[-\gamma(X-1)^{2} / 2\right],
$$

with

$$
X=E /\left\langle E^{2}\right\rangle^{1 / 2},
$$

since $I_{0}(Z)$ tends to $(2 \pi Z)^{-1 / 2} e^{Z}$, when $Z$ tends to infinity.

${ }^{1}$ M. Born and E. Wolf, Principles of Optics (Pergamon, London, 1970), 4th ed.

${ }^{2}$ C. Eckart, "The scattering of sound from the sea surface," J. Acoust. Soc. Am. 25, 566-570 (1953).

${ }^{3}$ P. Beckmann and A. Spizzichino, The Scattering of Electromagnetic Waves from Rough Surfaces (Pergamon, London, 1963).

${ }^{4} \mathrm{I}$. Tolstoy and C. S. Clay, Ocean Acoustics Theory and Experiment in Underwater Sound (McGraw-Hill, New York, 1966), Chap. 6.

${ }^{5}$ C. S. Clay and W. K. Leong, "Acoustic estimates of topography and roughness spectrum of the sea floor southwest of the Iberian Peninsula," in Physics of Sound in Marine Sediments (Plenum, New York, 1974), pp. 373-445.

${ }^{6}$ C. S. Clay and H. Medwin, Acoustical Oceanography: Principles and Applications (Wiley, New York, 1977), Chap. 10, Appen. 10.

${ }^{7}$ F. G. Bass and I. M. Fuks, Wave Scattering from Statistically Rough Surfaces (Pergamon, New York, 1979).

${ }^{8} \mathrm{~L}$. Brekhovskikh and Yu Lysanov, Fundamentals of Ocean Acoustics (Springer, New York, 1982), Chap. 9.

${ }^{9}$ P. Faure, "Theoretical model of reverberation noise," J. Acoust. Soc. Am. 36, 259-266 (1964)

${ }^{10}$ V. V. Ol'shevskii, Characteristics of Sea Reverberation (Plenum, New York, 1967).

${ }^{11} \mathrm{D}$. Middleton, “A statistical theory of reverberation and similar first-order scattered fields-Part I: Waveforms and general process; Part II: Moments, spectra and special distributions," IEEE Trans. Inform. Theory IT-13, 372-414 (1967).

${ }^{12}$ T. K. Stanton, "Sonar estimates of microroughness," J. Acoust. Soc. Am. 75, 809-818 (1984).

${ }^{13}$ C. de Moustier, "A Sea Beam acoustic data acquisition system," MPL TM-379, Marine Physical Laboratory, Scripps Institution of Oceanography, San Diego, CA (1985).
${ }^{14}$ Eckart's ${ }^{2}$ small-slope approximation was not used in this derivation. Had this approximation been used, the angular dependence in the fraction $B_{1}$ of Eq. (1) would appear as a $\cos \theta$ in the numerator instead of the denominator. The discrepancy between these two results comes from the contribution of the horizontal component of wavenumber $(a)$, which is neglected in the small-slope approximation.

${ }^{15} \mathrm{C}$. S. Clay, "Coherent reflection of sound from the ocean bottom," J. Geol. Res. 71, 2037-2046 (1966).

${ }^{16} \mathrm{~J}$. M. Proud, R. T. Beyer, and P. Tamarkin, "Reflection of sound from randomly rough surfaces," J. Appl. Phys. 31, 543-552 (1960).

${ }^{17} \mathrm{~J}$. C. Novarini and J. W. Caruthers, "The degree of coherence of acoustic signals scattered at randomly rough surfaces," J. Acoust. Soc. Am. 51, 417-418 (1972).

${ }^{18} \mathrm{C}$. W. Horton, "A review of reverberation, scattering, and echo structure," J. Acoust. Soc. Am. 51, 1049-1061 (1972).

${ }^{19}$ S. O. Rice, "Mathematical analysis of random noise Part III," Bell Syst. Tech. J. 24, 46-156 (1945).

${ }^{20} \mathrm{~J}$. W. Goodman, "Statistical properties of laser speckle patterns," in Laser, Speckle and Related Phenomena, edited by J. C. Dainty (Springer, New York, 1975), pp. 9-75.

${ }^{21} \mathrm{C}$. $\mathrm{L}$. Dolph, "A current distribution of broadside arrays which optimizes the relationship between beam width and side-lobe level," Proc. Inst. Radio Eng. 34, 335-348 (1946).

${ }^{22} \mathrm{~V}$. Renard and J. P. Allenou, "Sea Beam multi-beam echo-sounding in Jean Charcot Description, evaluation and first results," Internat. Hydrograph. Rev. Monaco LVI(1), 35-67 (1979).

${ }^{23}$ C. de Moustier and M. C. Kleinrock, "Bathymetric artifacts in Sea Beam: How to recognize them, what causes them," J. Geophys. Res. (in press).

${ }^{24}$ F. N. Spiess and P. F. Lonsdale, "Deep tow rise crest exploration techniques," Mar. Tech. 16, 67-75 (1982).

${ }^{25} \mathrm{~J}$. G. Kosalos and D. N. Chayes, "A portable system for ocean bottom imaging and charting," Proc. Oceans '83, 649-656 (1983).

${ }^{26} \mathrm{C}$. de Moustier, "Inference of manganese nodule coverage from Sea Beam acoustic backscattering data," Geophysics 50, 989-1001 (1985).

${ }^{27}$ W. R. Normark and D. J. W. Piper, "Sediments and growth pattern of Navy deep-sea fan, San Clemente basin, California borderland," J. Geol. 80, 198-223 (1972).

${ }^{28} \mathrm{~K}$. O. Emery, The Sea off Southern California-A Modern Habitat of Petroleum (Wiley, New York, 1960).

${ }^{29}$ Rise Axis Tectonic Team, "An along strike Sea Beam and Sea Marc I perspective of the axis of the East Pacific Rise: Implications for the accretion of oceanic lithosphere, "International Lithosphere Congress, Texas A\&M University (1983).

${ }^{30}$ R. J. Urick, Principles of Underwater Sound (McGraw-Hill, New York, 1983), 3rd ed.

${ }^{31}$ J. S. Bendat and A. G. Piersol, Random Data: Analysis and Measurement Procedures (Wiley, New York, 1971).

${ }^{32}$ T. K. Stanton, "Volume scattering: Echo peak PDF," J. Acoust. Soc. Am. 77, 1358-1366 (1985).

${ }^{33}$ A. Papoulis, Probability, Random Variables, and Stochastic Processes (McGraw-Hill, New York, 1965).

${ }^{34} \mathrm{C}$. W. Helstrom, Statistical Theory of Signal Detection (Pergamon, New York, 1968), 2nd ed. 